\title{
EBERHARD KARLS UNIVERSITÄT TÜBINGEN
}

FACUlty of SCIENCE - DePartment of Computer SCIENCE

\section{Validating Driver Profiles in the Daimler Traffic Simulation}

Author:

Fabian Dablander
Supervisor:

Prof. Dr. Martin Butz

First Examiner:

Prof. Dr. Martin Butz

Second Examiner:

Prof. Dr. Enkelejda Kasneci

A thesis submitted in fulfilment of the requirements

for the degree of Master of Science

$$
\text { in the }
$$

Chair of Cognitive Modeling

Department of Computer Science 


\section{Declaration of Authorship}

I, Fabian Dablander, declare that this thesis titled, "Validating Driver Profiles in the Daimler Traffic Simulation" and the work presented in it are my own. I confirm that:

- This work was done wholly while in candidature for a research degree at this University.

- Where any part of this thesis has previously been submitted for a degree or any other qualification at this University or any other institution, this has been clearly stated.

- Where I have consulted the published work of others, this is always clearly attributed.

- Where I have quoted from the work of others, the source is always given. With the exception of such quotations, this thesis is entirely my own work.

- I have acknowledged all main sources of help.

- Where the thesis is based on work done by myself jointly with others, I have made clear exactly what was done by others and what I have contributed myself.

Signed:

Date: 


\section{Abstract}

Driving simulators offer benefits such as a controlled, standardized, and thus replicable environment for experiments; ease of collecting highly accurate and precise measurements; and an overall high cost-effectiveness. To reap these benefits, however, driving simulation must be a valid measurement tool - behaviour observed in the simulation should generalize to the real road. Crucial for this is the behaviour of computer-generated drivers with which the driver interacts. The Daimler traffic simulation assumes, based on empirical studies, six distinct driver profiles. Does the behaviour of computer-generated drivers resemble human driving behaviour? Two experiments were conducted. In a simulation study, we tested whether we can recover the driver profile based on simulated driving data. While driver profiles were well separated in low and medium traffic scenarios, separation decreased in high traffic scenarios. These results are discussed and further studies suggested. In a driving simulator experiment, we tested whether the aggressive and calm driver profile improved upon an older version of the traffic simulation that lacked dedicated driver profiles. 32 participants had to judge whether the driver they encountered behaved like a human or computer, and had to provide realism judgements of its driving behaviour. Using Bayesian hierarchical regression methods, we found evidence that the driving behaviour of the aggressive driver is judged to stronger resemble human driving compared to the older version; other results were equivocal. I discuss these results and suggest improvements for future studies. Finally, in an online appendix, I discuss some of the exciting changes happening in scientific practice and culture. This includes a brief sketch of the "reproducibility crisis" in psychology, a tutorial on Bayesian statistics, and three practical recommendations for applied researchers. 


\section{Acknowledgements}

I want to thank everyone at the Daimler driving simulator who showed me that, hey, cool research happens outside of academia, too. On top of that, I was amazed by the excellent work and social environment. Thank you for being so kind.

I want to especially thank Florian Soyka, with whom I had the pleasure — the privilege to commute to work every day. Our discussions, which included topics ranging from causal entropic forces and philosophy of science to meaning and meditation, have enriched not only the last six months, but also my perspective on life.

Thanks to Steffi Preuß for always asking though questions and for extensive, critical, and sharp comments on this thesis. I cannot imagine the driving simulator without you. Thanks also to Christopher Kober, without whose work mine wouldn't exist, and who calmly hunted down bugs, even in times of great distress.

Peter Edelsbrunner, Jonas Haslbeck, Quentin Gronau, Michael Franke: you are a continuous reminder of the perks of academic life. Thank you.

Lastly, I want to thank my parents, my brother, and all members of my patchwork family who always supported me in my decisions, even in the whimsical ones. Somehow it all turned out okay. 


\section{Contents}

Declaration of Authorship ii

Abstract iii

Acknowledgements iv

1 Introduction 1

2 Simulation-based Validation $\quad 3$

2.1 Microscopic Traffic Models . . . . . . . . . . . . . . . . . . . . . . . 3

2.1.1 Modeling Driver Variability . . . . . . . . . . . . 5

2.2 Goal of this Research . . . . . . . . . . . . . . . . . . 9

2.2.1 Overview and Data . . . . . . . . . . . . . 10

2.3 Machine Learning . . . . . . . . . . . . . . . . . . . . . . . 10

2.3.1 Random Forests . . . . . . . . . . . . . . . . . . 13

2.3.2 Multidimensional Scaling . . . . . . . . . . . . . 16

2.4 Results . . . . . . . . . . . . . . . . . . . . . . 17

2.4.1 Prediction Accuracy . . . . . . . . . . . . . . . 17

2.4 .2 Clustering . . . . . . . . . . . . . . . 18

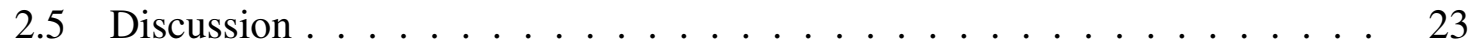

3 Human-based Validation $\quad 27$

3.1 Validity of Driving Simulations . . . . . . . . . . . . . . . . 27

3.2 A Note on Simulator Sickness _ . . . . . . . . . . . . . . 28

3.3 Goal of this Research . . . . . . . . . . . . . . . . . . . . . . . . . . . . . 29

3.4 Method . . . . . . . . . . . . . . . . . . . . . . 29

3.4.1 Participants . . . . . . . . . . . . . . . . 29

3.4 .2 Procedure . . . . . . . . . . . . . . . 30

3.4.3 Hypotheses and Statistical Rationale . . . . . . . . . . . . . . . . . . . 32

3.4 .4 Model Specification . . . . . . . . . . . . . . . . . 38

3.5 Results . . . . . . . . . . . . . . . . . . . 42

3.5.1 Confirmatory Hypothesis Testing . . . . . . . . . . . . . . 42

3.5.2 Exploratory Analysis . . . . . . . . . . . . . . . . . . . 43

3.5.3 Qualitative Results . . . . . . . . . . . . . . . . . 47

3.6 Discussion . . . . . . . . . . . . . . . . . . . . . . . . . . 49

$\begin{array}{ll}\text { References } & 55\end{array}$ 


\section{List of Figures}

2.1 Characteristics of an agent in a microscopic traffic model, based on Figure 2 of Kesting, Treiber, and Helbing (2009). . . . . . . . . . . . . . . . . . .

2.2 Shows how to generate an agent in the Daimler traffic simulation. Figure 2.3 and Figure 2.4 show the steps in the boxes. Main parameters are Speed, Safety, and Consideration. . . . . . . . . . . . . . . . . 7

2.3 Classification parameter divided into three categories . . . . . . . . . 8

2.4 Shows how to arrive at the parameter values given the value sampled from the uniform distribution for each of three possible mappings . . . . . . . . 9

2.5 Distribution of speed, acceleration, headway, and time on lane for one dataset across all driver profiles . . . . . . . . . . . . . . . . . 11

2.6 Displays the relationship between traffic density and traffic flow (left), and between traffic density and speed (right) . . . . . . . . . . . . . 12

2.7 Example of a classification tree using the CART algorithm predicting the flower species based its features using the famous Iris dataset (Anderson, 1936; Fisher, 1936) . . . . . . . . . . . . . . . . . .

2.8 Shows violin plots for the number of lane changes for each dataset and each driver profile in high traffic . . . . . . . . . . . . . . . . 18

2.9 Shows the mean decrease in Gini impurity for all variables for each of the steps in leave-one-out cross-validation on the light traffic simulation data . . 19

2.10 Shows the mean decrease in Gini impurity for all variables for each of the steps in leave-one-out cross-validation on the medium traffic simulation data

2.11 Shows the mean decrease in Gini impurity for all variables for each of the steps in leave-one-out cross-validation on the high traffic simulation data . .

2.12 Shows the similarity between the driver profiles based on a multidimensional scaling analysis of the proximity matrix returned by random forests . 22

3.1 Overview of the driving simulator study . . . . . . . . . . . . . 31

3.2 Shows how the Cauchy prior on $\beta$, which is the coefficient of the $0 / 1$ coded predictor $x$, influences the choice probability . . . . . . . . .

3.3 Shows mean and standard deviation of the proportion judged human for the respective driver profiles . . . . . . . . . . . . . . . . . . 43

3.4 Displays realism ratings across driver profiles . . . . . . . . . . . . . 44

3.5 Shows the Bayes factors in favour of the directed hypotheses for different Cauchy prior widths . . . . . . . . . . . . . . . . . 45 
3.6 Shows the mean and $95 \%$ credible intervals for the posterior distribution of predictors in the full logistic and full ordinal regression models, respectively. Note that continuous predictors have been scaled by subtracting the mean and dividing by twice the standard deviation (Gelman, 2008). profile_aggr, profile_calm, and scenario are dummy coded variables indicating the conditions; mean_presence.z is the average score in the presence questionnaire; gender is a dummy coded variable indicating gender $(0=$ female); sum_social...z are sums of the two social desirability subscales; $k m \_h i g h w a y \_d r i v e n . z$ are the kilometers that were driven on highways the previous year; pre_ssq.z is the simulator sickness score in the preliminary interview; $s d($ int $)$ is the standard deviation of the participant-specific offsets to the intercept. $\ldots \ldots \ldots \ldots \ldots$

3.7 Shows mean and standard deviation of the proportion judged human for the for each scenario 


\section{List of Tables}

2.1 Displays a short description of the driver profiles and their frequencies based on the German-Swiss studies conducted between 1972 - 1993. . . . . . . . 5

2.2 Overview of the studies that form the basis for the six driver profiles. Duration denotes the duration time or kilometres driven during the examination.

2.3 Parameters of the driver models used in the Daimler traffic simulation. Parameters with a (log) normal distribution have been empirically calibrated; linear parameters are set by IDM and MOBIL. . . . . . . . . . . . . . . .

2.4 Shows the categorisation of parameters into three categories for each driver profile. . . . . . . . . . . . . . . . . 8

$3.1 \quad$ Age and sex of participants $(N=32) \ldots \ldots \ldots \ldots$

3.2 Evidence categories for the Bayes factor $B F_{10}$. From Table 4 in Vandekerckhove, Matzke, and Wagenmakers (2015). . . . . . . . . . . . . 38 


\section{Chapter 1}

\section{Introduction}

Driving is one of the most complex tasks humans do on a frequent basis; it requires sensory, perceptual, cognitive, and motor functions which are influenced by a variety of environmental and personal factors. Thus, it should come as no surprise that driving research is inherently inter- and multidisciplinary, with applications ranging from medicine and psychology to engineering and computer science (Fisher, Rizzo, Caird, \& Lee, 2011).

Much of driving research employs driving simulators, which are machines of extreme technical complexity (for an overview, see Carsten \& Jamson, 2011; Schöner \& Morys, 2015). They hold considerable benefits: a controlled, standardized, and thus replicable environment for experiments; ease of collecting highly accurate and precise measurements on a variety of variables such as headway, lane position, and reaction time; the possibility of exposing drivers to dangerous situations without them being physically at risk; an excellent means to test novel user interfaces, driving assistance systems, and the dynamics of the vehicle before production; and an overall high cost-effectiveness (De Winter, Van Leuween, \& Happee, 2012).

In order to provide useful data, however, driving simulators must yield valid measurements (e.g., Klüver, Herrigel, Heinrich, Schöner, \& Hecht, 2016). Validity refers to the extent to which behaviour observed in the simulator can be generalized to the real road. There are many aspects influencing validity, ranging from the physical — which car and what environment? - to the psychological — does this feel like acceleration? An important aspect is the behaviour of other drivers in the simulation. For example, when testing a new assistance system that aids the driver in reacting to potentially dangerous situations, 
other drivers must behave realistically in order to generalize the behaviour observed in the simulation to the real world.

The goal of this thesis is to take first steps towards evaluating the validity of the Daimler traffic simulation which assumes six distinct driver types based on studies conducted in the 1970s, 1980s, and 1990s (Hürlimann, 1996). The first chapter focuses on a simulation-based validation. In it, I provide an overview of traffic modeling more broadly, discuss how the Daimler traffic simulation works, and test whether one can recover the driver profiles using machine learning techniques on simulated data. I critically discuss the results, scrutinize the empirical basis of the six driver profiles, suggest practical steps towards improving the traffic simulation, and propose ideas for future studies.

Do the empirically based driver profiles improve upon an older version of the traffic simulation which lacked distinct driver profiles? How do participants experience the traffic simulation as a whole? To answer these questions, a driving simulator experiment has been conducted. Chapter 3 describes this experiment and critically reflects its results.

Scientific research in an applied industry context differs markedly from scientific research in an academic context. It is my experience that academic researchers, if not apply, at least discuss novel methods and reflect on their behaviour more frequently, while applied researchers rely more heavily on trusted tools and are slower to adopt new methodologies. This makes sense. In industry, the wheel of profits must keep on turning, and pressure from external stakeholders prevent deeper reflection whereas academia provides more freedom and time to critically reflect on dominant ideas and practices. Currently, there is a revolution going on in psychology, the social and biomedical sciences more broadly — a paradigm shift with respect to the way scientific research is funded, planned, executed, and communicated. On online appendix to this thesis is dedicated to bringing these issues and developments closer to the applied researcher. In addition to that, it serves as a basic introduction to Bayesian statistics and provides three practical recommendations to increase the validity and reproducibility of applied research. 


\section{Chapter 2}

\section{Simulation-based Validation}

\subsection{Microscopic Traffic Models}

Traffic is a multi-agent system in which drivers operate and react to each other in shared infrastructure (Kesting, Treiber, \& Helbing, 2009). Broadly speaking, there are two modeling perspectives on such a system. Macroscopic traffic models view traffic as a physical flow of a fluid and describe it in terms of aggregated quantities such as average velocity, traffic density, and traffic flow (e.g., Helbing, Hennecke, Shvetsov, \& Treiber, 2001). Microscopic traffic models, on the other hand, are agent-based, focusing on the behaviour of each individual driver, such as acceleration, breaking, and lane changes (Kesting et al., 2009; van Wageningen-Kessels, Van Lint, Vuik, \& Hoogendoorn, 2015).

Agents in microscopic traffic models have internal features that are characteristic of human drivers as well as external features that depend on the vehicle and its environment. Figure 2.1 shows both internal and external dimensions of the driving behaviour. Each dimension has operational and strategic aspects that determine the driver's behaviour. For example, an internal, strategic aspect would be the driver's desire to move with a certain velocity, while an external, strategic aspect might describe the laws and regulations of traffic itself, as instantiated, for example, by speed limits. In contrast, operational aspects are less goal oriented and influence the driving behaviour at the moment. Reaction time and a limited attention span, for instance, are internal operational aspects while road conditions such as snow or rain would count as external operational conditions. The task put to agent-based traffic models is to appropriately account for these aspects in order to realistically simulate real-world driving behaviour (Kesting et al., 2009). 
FIGURE 2.1: Characteristics of an agent in a microscopic traffic model, based on Figure 2 of Kesting, Treiber, and Helbing (2009).

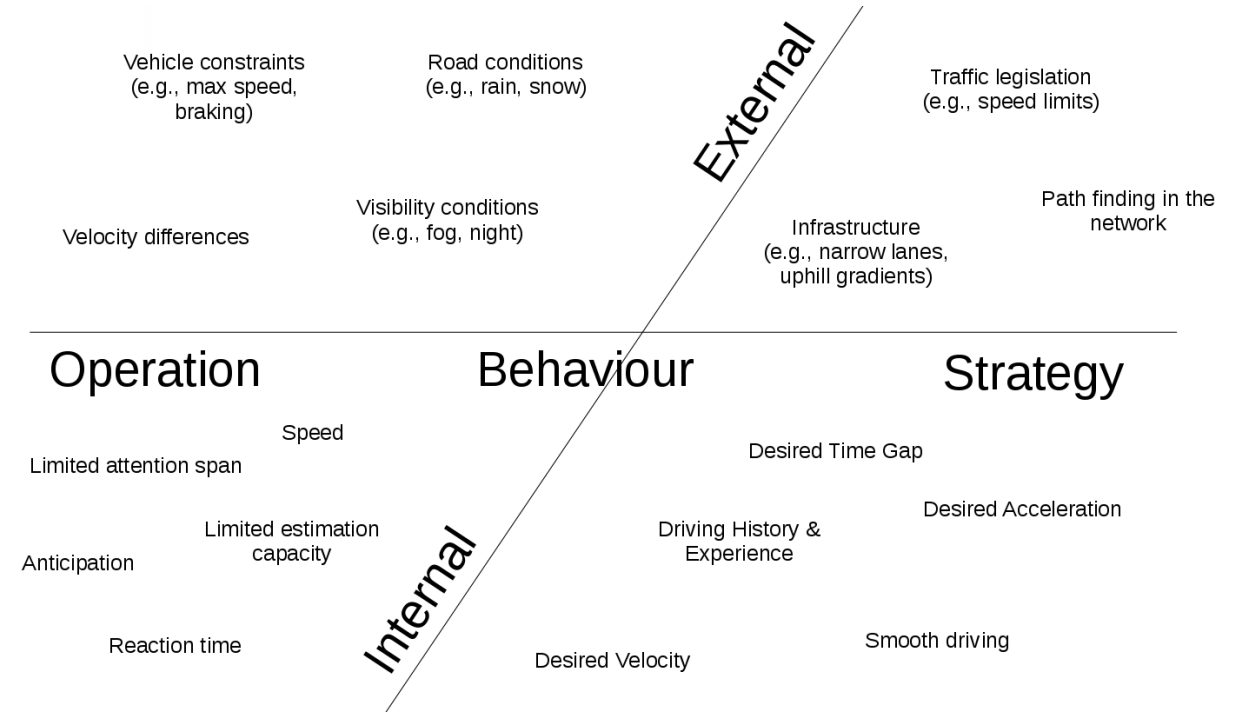

Every microscopic traffic model describes the longitudinal (car-following) and lateral (lanechanging) behaviour of agents (cf., van Wageningen-Kessels et al., 2015; Kesting et al., 2009, p. 7). The Intelligent Driver Model (IDM) is a popular approach (Treiber, Hennecke, \& Helbing, 2000) to modeling longitudinal movements. It belongs to the class of deterministic car-following models, and it is formulated as an ordinary differential equation (for a detailed description, see Kesting et al., 2009). The MOBIL (minimizing overall braking induced by lane changes) algorithm models lane-changes by comparing the potential gain in acceleration at the other lane with the acceleration at the current lane and, based on this criterion, decides whether to change lanes (Kesting, Treiber, \& Helbing, 2007).

With the IDM and MOBIL, basic driving behaviour can be modelled. People differ in their driving behaviour, however: some have an inherent tendency to drive faster, spending most of their time on the leftmost lane, while others are more considerate, avoid speeding, and spend their time mostly on the right lane. An important challenge for traffic simulations is to account for these differences. 
TABLE 2.1: Displays a short description of the driver profiles and their frequencies based on the German-Swiss studies conducted between 1972 - 1993.

\begin{tabular}{llll}
\hline Profile & $\%$ Men & $\%$ Women & Description \\
\hline calm & 27 & 42 & foresightful, considerate \\
active & 32 & 30 & fun, fast \\
sporty & 15 & 10 & thrill, increased velocity \\
affective & 11 & 9 & unconcentrated, frantic \\
insecure & 6 & 6 & hesitant, poor reaction \\
aggressive & 9 & 3 & consciously obstructive, markedly too fast \\
\hline
\end{tabular}

\subsubsection{Modeling Driver Variability}

To account for the fact that humans vary in their driving behaviour, parameters in traffic simulations can be set differently across drivers. For example, aggressive and insecure drivers might get assigned a DesiredVelocity of $140 \mathrm{~km} / \mathrm{h}$ and $100 \mathrm{~km} / \mathrm{h}$, respectively. To avoid setting parameters arbitrarily, actual driving data can be used to calibrate them. The modeling approach by Christopher Kober and Daimler relies on data gathered from 266 employees that were given a Mercedes-Benz car to test drive it, yielding about 25 terrabytes of data on speed, acceleration, lane changes, etc. Moreover, the parameter specification is based on empirical literature which postulates six distinct driver profiles.

\section{Empirical Foundation}

The traffic simulation under discussion assumes six driver profiles that differ in their driving behaviour. They are based on empirical studies conducted by the Institut für Angewandte Psychologie Zürich in cooperation with the TÜV Akademie Bayern through the years 1972 - 1992 and 1998 (Flade \& Limbourg, 1999; Jöri, 2002, ch. 6). Table 2.1 lists the driver profiles, their frequency of occurrence, and a short description. In total, 7.821 men and 2.736 women were tested across five studies that had different goals and which used different measures (Hürlimann, 1996, p. 67-106), see Table 2.2. 
TABLE 2.2: Overview of the studies that form the basis for the six driver profiles. Duration denotes the duration time or kilometres driven during the examination.

\begin{tabular}{|c|c|c|c|c|c|}
\hline Study & $\mathrm{N}$ & Years & Duration & Measurements & Purpose \\
\hline MIKRO & 1042 & 1972-1974 & $6.4 \mathrm{~km}$ & $\begin{array}{l}\text { observations (speed, lateral- \& longi- } \\
\text { tudinal acceleration), interviews (at- } \\
\text { tidutes, background) }\end{array}$ & $\begin{array}{l}\text { collect information about the introduc- } \\
\text { tion of a } 130 \mathrm{~km} / \mathrm{h} \text { speed limit on high- } \\
\text { ways compared to } 100 \mathrm{~km} / \mathrm{h} \text { inner city }\end{array}$ \\
\hline SIDI & 117 & 1987-1988 & $47.6 \mathrm{~km}$ & $\begin{array}{l}\text { aptitude tests, personality test, psy- } \\
\text { chophysiological measurements }\end{array}$ & $\begin{array}{l}\text { investigate the effect of the position } \\
\text { and frequency of traffic signs on driv- } \\
\text { ing behaviour }\end{array}$ \\
\hline KRD & 5048 & 1972-1992 & $30 \mathrm{~min}$ & tests for motorists by the TÜV Bayern & $\begin{array}{l}\text { examine the aptitude of motorists that } \\
\text { transport commodities for a living }\end{array}$ \\
\hline AUTOP & 2242 & 1986 & $9.5 \mathrm{~km}$ & $\begin{array}{l}\text { observations (speed, lateral- \& longi- } \\
\text { tudinal acceleration) }\end{array}$ & $\begin{array}{l}\text { examine potential driving differences } \\
\text { between men and women. }\end{array}$ \\
\hline ÖKO & 2108 & 1990-1993 & $50 \mathrm{~km}$ & $\begin{array}{l}\text { tests for motorists by the TÜV Bayern, } \\
\text { fuel consumption }\end{array}$ & $\begin{array}{l}\text { examine the aptitude of motorists that } \\
\text { transport commodities for a living as } \\
\text { well as measure the efficiency of their } \\
\text { driving behaviour }\end{array}$ \\
\hline
\end{tabular}

Based on the data gathered from "MIKRO" at the beginning of the 1970s, six distinct driver profiles were developed (Hürlimann, 1996, p. 74). Data from future studies were analysed and categorized according to the six profiles. For example, taking the calm driver as a benchmark, they "AUTOP" study found that $47 \%$ of the aggressive drivers and $43 \%$ of the affective drivers drift more than $20 \mathrm{~cm}$ to the left of the lane; using data from "MICRO", "SIDI", and "AUTOP", they found that affective, sporty, and aggressive drivers have an increased velocity of $2 \%, 6 \%$ and $11 \%$, respectively (Hürlimann, 1996, p. 78$)^{1}$. Other findings pertain to traffic law violations, attitudes towards safety restrictions such as seat belts, sensitivity to dangerous situations, personality, and psychophysiological measurements (Hürlimann, 1996, p. 79-106).

\section{Software Implementation}

In the Daimler traffic simulation, the driver profiles differ across the dimensions Speed (five parameters), Safety (two parameters), and Consideration (two parameters); see Table 2.3 for an overview of the parameters, their units, distribution, empirically estimated mean and

\footnotetext{
${ }^{1}$ These are all descriptive statistics. Throughout, Hürlimann (1996) avoids using inferential statistics. I will return to this point in the discussion.
} 
TABLE 2.3: Parameters of the driver models used in the Daimler traffic simulation. Parameters with a (log) normal distribution have been empirically calibrated; linear parameters are set by IDM and MOBIL.

\begin{tabular}{lllll}
\hline Parameter & Unit & Distribution & Mean & SD \\
\hline Speed & & & & \\
DesiredVelocity & $\mathrm{km} / \mathrm{h}$ & normal & 142.89 & 29.72 \\
LaneChangeTime & $\mathrm{s}$ & normal & 8.00 & 2.25 \\
maxComfLongAcceleration & $\mathrm{m} / \mathrm{s}^{2}$ & log normal & -0.24 & 0.24 \\
maxComfLongDeceleration & $\mathrm{m} / \mathrm{s}^{2}$ & log normal & 0.25 & 0.29 \\
LaneChangeThreshold & $\mathrm{m} / \mathrm{s}^{2}$ & linear & 0.30 & 0.20 \\
Safety & & & & \\
$\begin{array}{l}\text { DesiredHeadway } \\
\text { minGapFront }\end{array}$ & $\mathrm{S}$ & log normal & 0.16 & 0.4 \\
Consideration & $\mathrm{m}$ & linear & 1.5 & 1 \\
politenessNorm & & & & \\
RightLaneBias & $\mathrm{normalized}$ & linear & 0.5 & 0.5 \\
\hline & $\mathrm{m} / \mathrm{s}^{2}$ & linear & 0.3 & 0.4 \\
\hline
\end{tabular}

standard deviation. For each driver profile and parameter, three groups are created based on discretizing a uniform distribution into three pieces, see Figure 2.3; an overview of the groups across parameters is displayed in Table 2.4.

FIGURE 2.2: Shows how to generate an agent in the Daimler traffic simulation. Figure 2.3 and Figure 2.4 show the steps in the boxes. Main parameters are Speed, Safety, and Consideration.

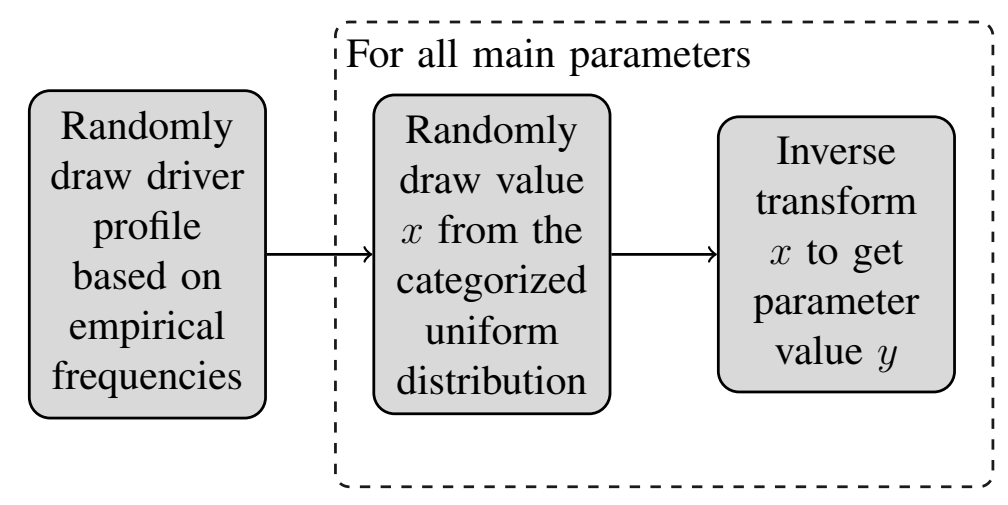

How is a particular agent generated? Figure 2.2 provides an overview. First, a driver profile is randomly drawn based on the empirical frequency distribution of profiles as posited in the literature. For each main parameter, a value $x$ is drawn from a uniform distribution with 


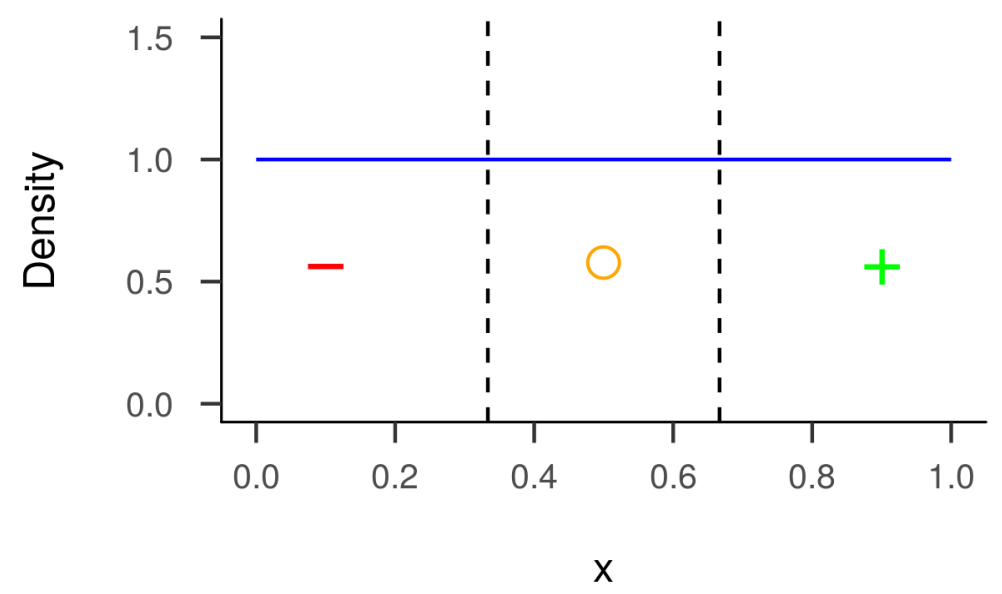

FIGURE 2.3: Classification parameter divided into three categories.

particular limits. Then, depending on the distribution of the specific parameter, $x$ is inverse transformed into the parameter value $y$.

TABLE 2.4: Shows the categorisation of parameters into three categories for each driver profile.

\begin{tabular}{lcccccc}
\hline Parameter & calm & active & sporty & affective & insecure & aggressive \\
\hline Frequency & $35 \%$ & $31 \%$ & $11 \%$ & $11 \%$ & $6 \%$ & $6 \%$ \\
Speed & 0 & + & + & 0 & - & + \\
Safety & + & + & - & - & + & - \\
Consideration & + & + & 0 & - & + & - \\
\hline
\end{tabular}

To illustrate, assume an aggressive driver and the features DesiredVelocity, MaxComfLongAcc, and politenessNorm. With respect to the first two, the aggressive driver is in the "+" group; thus values can be in the range $\left[\frac{2}{3}, 1[\right.$. Upon uniformly drawing a random value in this range, say $x=.69$, we arrive at a desired velocity of $y=157.63$ by inverse transforming the Gaussian distribution associated with speed (see Table 2.3), and $y=-.12$ for the (log) maximal longitudinal acceleration that the driver is still comfortable with, by inverse transforming the particular lognormal distribution; see Figure 2.4. Politeness is in a different category, so a new random value is generated. For the aggressive driver, politeness is in the “-" group; thus values can be in the range $] 0, \frac{1}{3}[$. After drawing a value $x=.2$, we arrive at a politenessNorm of $y=.6$ by checking the linear function associated with politeness; see 
Figure 2.4.
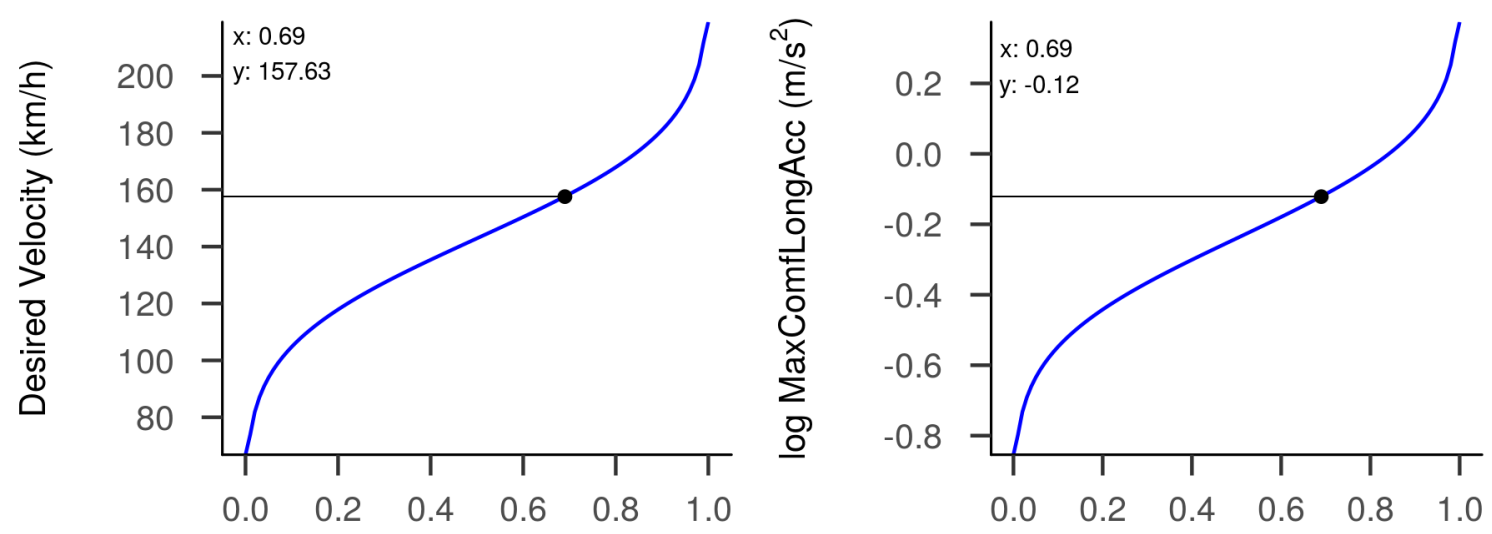

$\mathrm{X}$

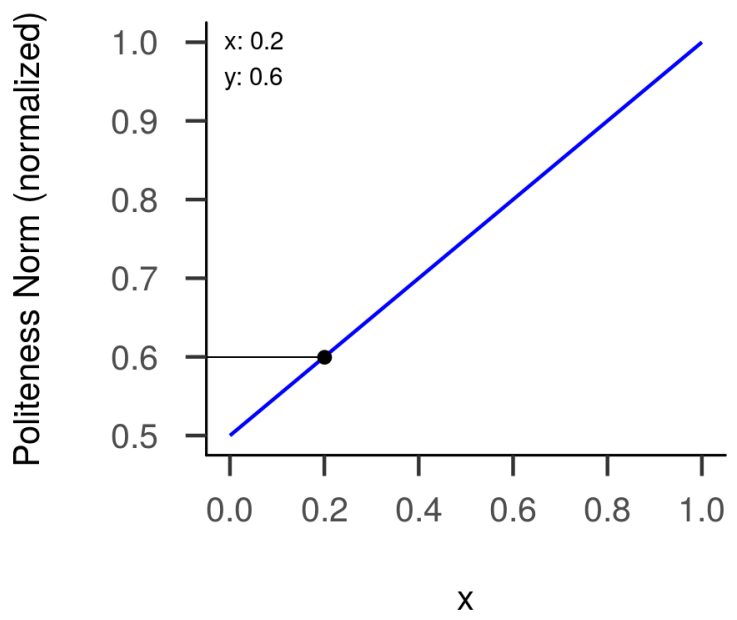

FIGURE 2.4: Shows how to arrive at the parameter values given the value sampled from the uniform distribution for each of three possible mappings: normal, log normal, linear.

\subsection{Goal of this Research}

The traffic model assumes six distinct driver profiles and describes a mechanism of generating them. Given simulated traffic data, can we recover these driver profiles? That is, are they sufficiently distinct that one can distinguish them based on objective features such as speed and headway? 
Answering this question is a first, basic step in model validation. It is completely internal and does not require real world benchmarks. Other means of validating traffic simulations entail comparing the emergent properties of the resulting traffic, such as the occurrences of traffic jams and the distribution of cars across lanes (e.g., Cristea, Rulewitz, Radusch, Hübner, \& Schünemann, 2016), or measuring how the system changes when changing certain parameters (e.g., Nourzad, Salvucci, \& Pradhan, 2014).

\subsubsection{Overview and Data}

For each driver profile, we simulated 50 datasets consisting of 75.9 kilometres of driving on a three lane highway with ten observations per second in low $(\rho=15)$, medium $(\rho=36)$, and high traffic $(\rho=63)$; see Figure 2.6 for the relation between traffic flow and speed in the Daimler traffic simulation. We observed the individual driver profiles, who were surrounded by traffic in which all driver types occurred, with respect to speed, acceleration, headway, number of lane changes, and time spent on each lane; for a visualization of one such dataset, see Figure 2.5. For each dataset and driver profile, we summarized the distribution of speed, headway, and acceleration by the deciles of the empirical histogram ${ }^{2}$. We tested whether we could predict the driver profile based on these summary measures of the datasets using random forests with leave-one-out crossvalidation. Additionally, we visualized the similarity across driver profiles using multidimensional scaling. All analyses were carried out using the R statistical computing language (R Core Team, 2017).

\subsection{Machine Learning}

We focus on supervised learning here. Let $X \in \mathbb{R}^{p}$ be a real valued random input variable and $Y \in \mathbb{N}$ an integer valued random output variable with joint distribution $p(X, Y)$. The

\footnotetext{
${ }^{2}$ Thus, for example, the variable speed describing the histogram gets cut into the single values speed00, speed $10, \ldots$, speed 100.
} 

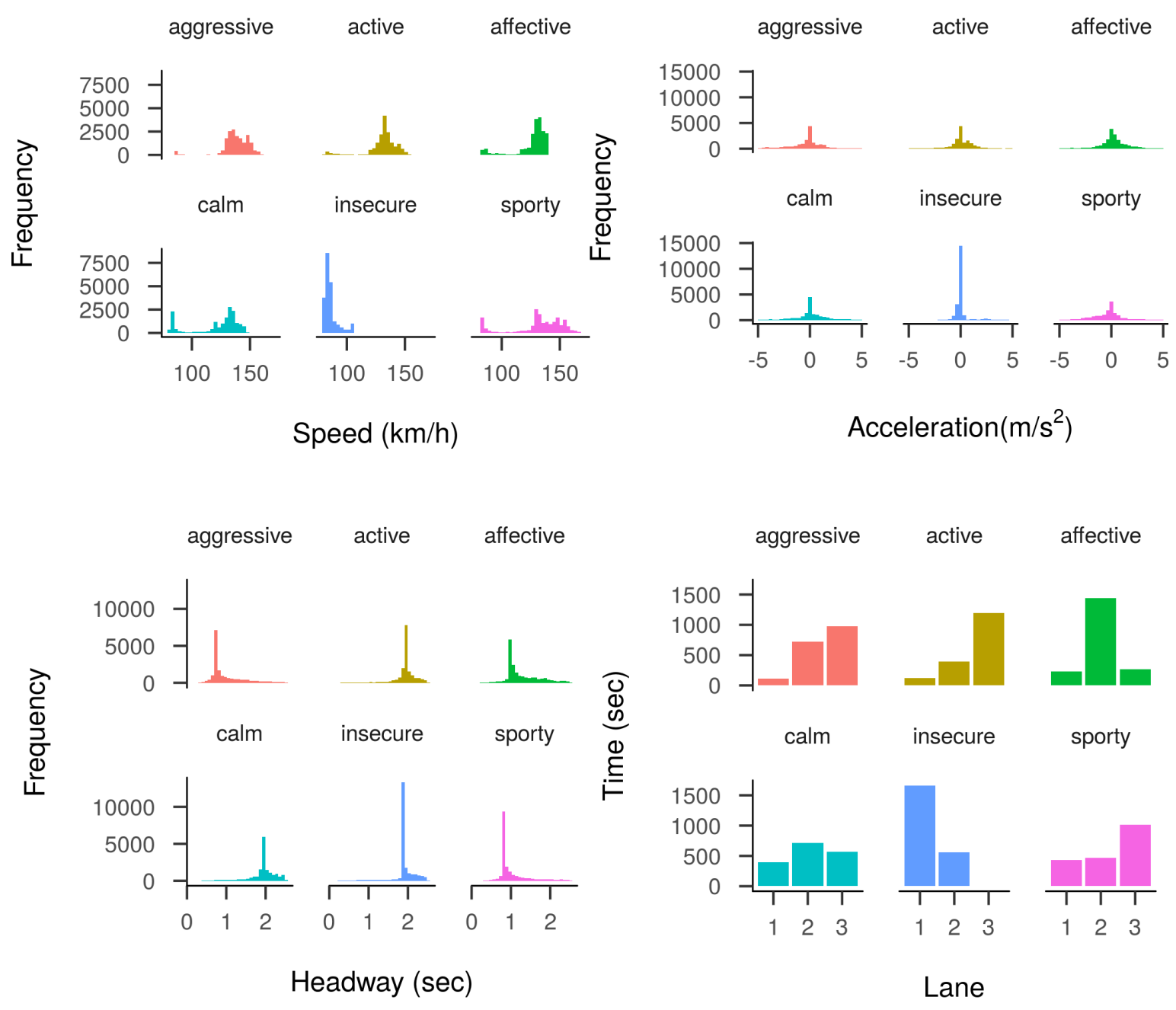

FIGURE 2.5: Distribution of speed, acceleration, headway, and time on lane for one dataset across all driver profiles. 


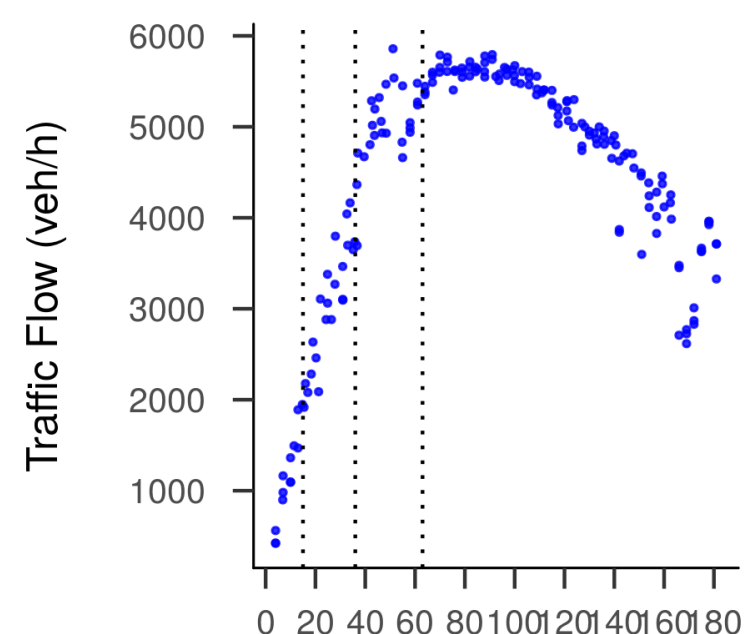

Density $(\mathrm{veh} / \mathrm{km})$

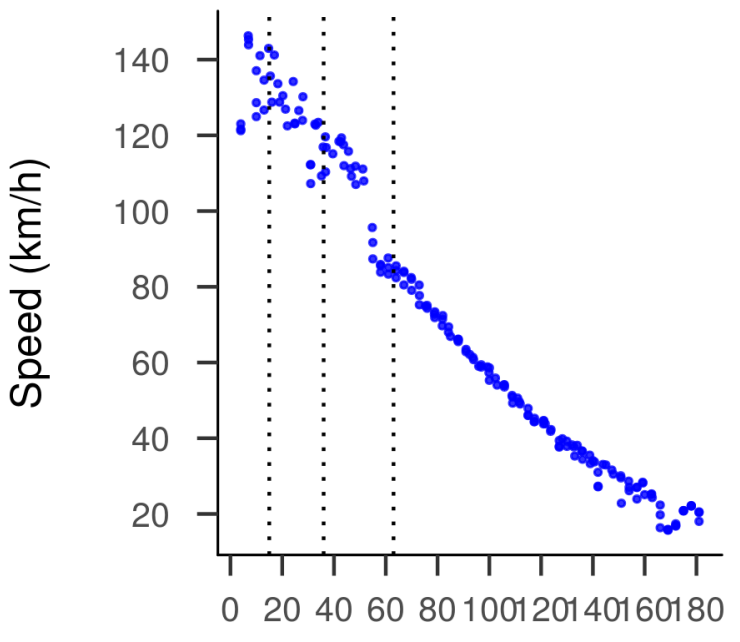

Traffic Density (veh/km)

FIGURE 2.6: Displays the relationship between traffic density and traffic flow (left), and between traffic density and speed (right). Dotted lines indicate parameter traffic density settings we have used.

goal of supervised machine learning is to find a function $f \in \mathcal{F}$ for classifying $Y$ given the input $X$. A loss function $\mathcal{L}$ describes how well a particular $f$ achieves this goal. This leads us to a criterion for choosing $f$, namely the expected prediction error

$$
\mathrm{EPE}=\mathbb{E}[\mathcal{L}(f(X), Y)]
$$

where the expectation is taken with respect to the joint distribution $p(X, Y)$, that is, practically speaking, averaging over all possible data sets (Hastie, Tibshirani, \& Friedman, 2009). Because we do not have all possible datasets, we can only approximate the expected prediction error; to achieve this, we use cross-validation.

The landscape of different $f$ is vast. In this research, we focus on random forests (e.g., Breiman, 2001; Strobl, Malley, \& Tutz, 2009; Cutler et al., 2007; Liaw \& Wiener, 2002) because of their good predictive accuracy and their capacity to construct a proximity matrix that indicates the similarity across the driver profiles (e.g., Liaw \& Wiener, 2002, p. 20-21); but more on that below. 


\subsubsection{Random Forests}

Random forests are ensemble learning methods. An ensemble learning method aggregates over many weak learners to improve predictive accuracy (e.g., Efron \& Hastie, 2016, ch. 17). In the case of random forests and our classification problem, the weak learner is a single classification tree. Classification trees create binary splits in the data in order to construct increasingly homogeneous groups. A particular split point of a predictor results in new branches of the tree. To illustrate, consider the famous Iris data set which describes how three species of flowers - setosa, versicolor, virginica - differ in their petal and sepal length and width (Anderson, 1936; Fisher, 1936). Figure 2.7 demonstrates how a classification tree arrives at a decision rule or function $f$ that separates these species of flowers. Splitting petal length at the value 2.5 leads to a complete isolation of setosa. The node is now fully homogeneous and does not require further splitting. The other two flowers are separated by a petal width of 1.8. Flowers that have a petal length above 2.5 but a petal width below 1.8 are categorized as versicolor. The rest are classified as virginica. The bottom plot in Figure 2.7 displays the piecewise constant decision boundaries and shows that five flowers of species virginica are misclassified as versicolor.

There exist a number of classification tree algorithms that differ chiefly in the way they split predictor variables. For example, $C 4.5$ uses entropy to measure how homogenous groups are, while CART uses a generalization of the binomial variance called the Gini index (Loh, 2011, p. 14).

Two major issues are associated with classification trees (see Strobl et al., 2009, p. 330-331). First, they are rather instable, that is, they emit high variability. This is because the splitting criterion depends strongly on the particular observations in the training set. Small changes in these observations can change the splitting criterion, and because nodes higher up the tree influence lower nodes, a tree constructed from a particular dataset might end up looking very different from a tree constructed from a similar dataset, even if the data generating process 


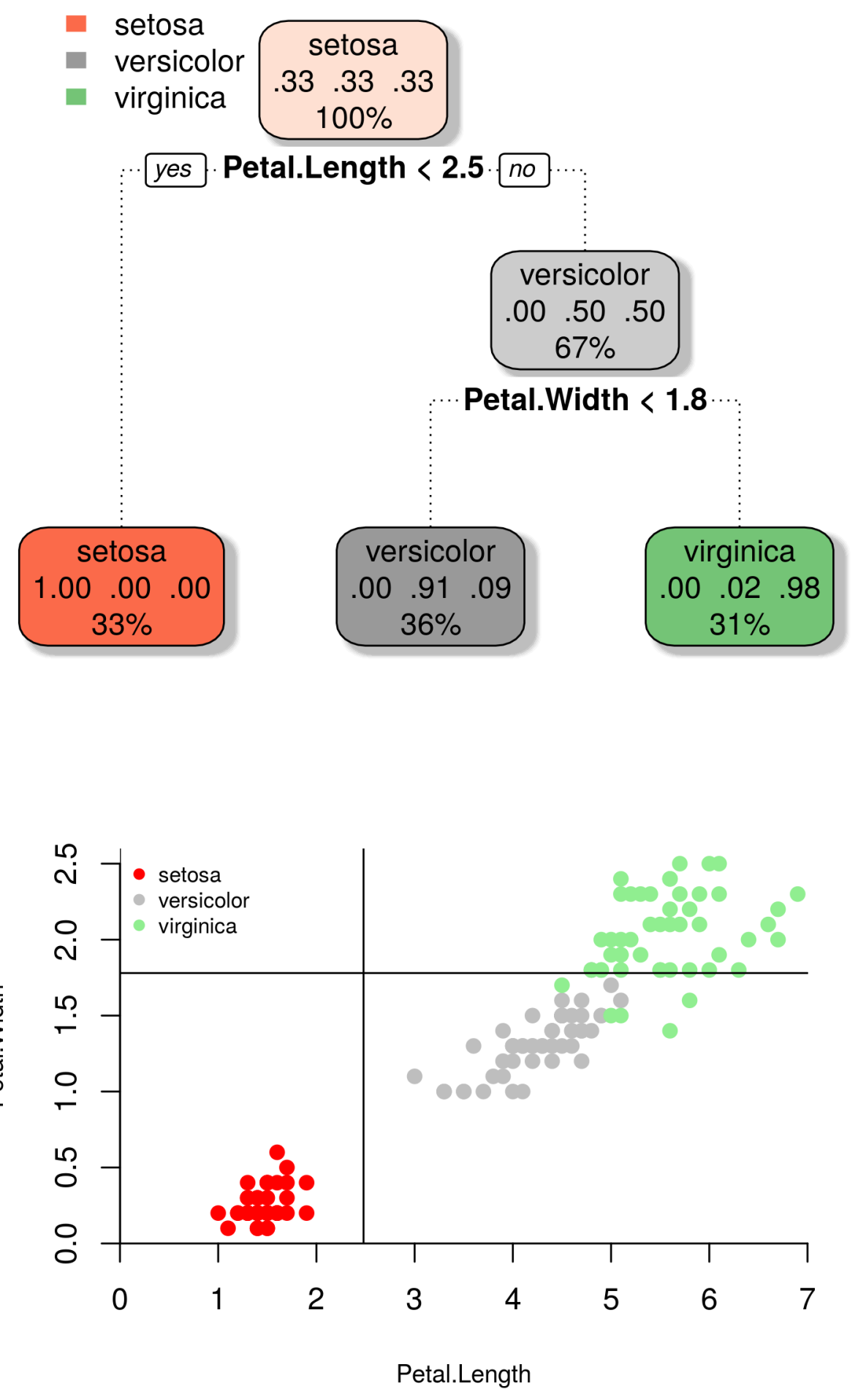

FIGURE 2.7: Example of a classification tree using the CART algorithm predicting the flower species based its features using the famous Iris dataset (Anderson, 1936; Fisher, 1936). Top plot displays the decision tree, bottom plot the decision regions. 
was the same in both cases ${ }^{3}$. Second, the prediction of single trees is piecewise constant which can lead to jumps in the particular predictions; small changes in the predictor values can have large effects on the resulting classification.

Ensemble learning methods avoid these two issues by basing predictions on many trees instead of only a single one. Both bagging and random forests are such methods. In these approaches, either a bootstrap sample or a subset is drawn randomly from the training data. An individual tree is grown on these data, and at the end of the procedure predictions are made by averaging over all single trees (Efron \& Hastie, 2016, ch. 17). Random forests add another layer of randomness. At each split, only a randomly selected subset of the predictor variables are considered, leading to even more diverse individual trees (Strobl et al., 2009, p. 333). (Bagging is a special case of random forests where the number of randomly selected predictor variables equals the total number of predictor variables.) By averaging over many single trees (weak learners), the piecewise constant decision boundaries become smooth. Additionally, predictions become more stable because these ensemble methods equalize the effects of particular observations.

Random forests only require the specification of two parameters: the number of trees we want to grow, and the number of predictor variables that are randomly chosen for the construction of the individual classification trees. I have set the number of trees to 2000 in order to gain stable estimates of variable importance and proximity, and left the number of predictor variables at the default of $\sqrt{p}$, where $p$ is the number of variables (Liaw \& Wiener, 2002).

In the Iris classification example, petal length and petal width suffice for accurate classification; they are the most important variables. How do random forests construct indices of variable importance? Such indices are based on a decrease in node impurity as measured by the Gini coefficient, and are — technically speaking — a by-product of training the random

\footnotetext{
${ }^{3}$ This can be demonstrated by building classification trees on bootstrap samples of the data, see Figure 5 in Strobl et al. (2009).
} 
forest. The Gini coefficient was initially proposed as a summary measure of income inequality in a society (Gini, 1912), but can be used more generally as a measure of sparseness, for example to evaluate deep neural network layer representations (Guest, 2017). For details on how it is used by the random forest algorithms, see e.g., Menze et al. (2009, p. 2-3).

\subsubsection{Multidimensional Scaling}

In contrast to prediction problems, clustering is about finding similar groups, called clusters, in the $p$-dimensional feature space carved out by $X$ (Hennig, 2015). Incidently, random forests can also be used for clustering (Liaw \& Wiener, 2002, p. 20-21). To do so, note that observations which are categorized in the same terminal node are more similar to each other. After all, this is exactly what classification trees are all about: creating homogeneous groups based on splitting predictor variables. This fact allows the construction of a proximity matrix where the element $(i, j)$ denotes the fraction of trees in which observations $i$ and $j$ fall in the same terminal node.

What is the best coordinate system to describe this proximity matrix? Or, put another way, how can we best visualize the similarity between the observations? Multidimensional scaling (MDS) provides an answer (see Hout, Papesh, \& Goldinger, 2013, for applications of MDS in cognitive science). Let $\delta_{i j}$ be the distance between elements $i$ and $j$. The idea behind MDS is to find a set of vectors $x_{1}, \ldots, x_{k}$, where $k$ is the number of desired dimensions, that describe a coordinate system in which these distances are maintained. MDS is usually formulated as an optimization problem where the set of vectors is found by minimizing some cost function that depends on the particular distance metric employed (for an overview, see Steyvers, 2002; Wickelmaier, 2003). Here, we use two-dimensional ${ }^{4}$ classical $^{2}$ multidimensional scaling, which relies on Euclidean distances; because Euclidean distances

\footnotetext{
${ }^{4}$ While a three-dimensional solution fits slightly better, we prefer two-dimensions because they are easier to visualize. Note that conclusions drawn based on the visualization to not change either way.
} 
are invariant to translation, rotation, and reflection, the resulting coordinate system, consisting of vectors $x_{1}$ and $x_{2}$, is invariant to them, too.

\subsection{Results}

Figure 2.5 displays the distribution of speed, acceleration, headway, and time one the lanes for each driver profile for a particular dataset. We find that the insecure driver rarely drives faster than $100 \mathrm{~km} / \mathrm{h}$, that he holds large headway, and that he spends most of his time on the leftmost lane. In contrast, the affective driver drivers much faster, holds less headway, and spends most of his time on the middle lane. How well can we separate those, and other, driver profiles?

\subsubsection{Prediction Accuracy}

We used leave-one-out cross-validation to estimate the prediction accuracy of random forests for low ( $\rho=15)$, medium $(\rho=36)$, and high $(\rho=63)$ traffic scenarios. That is, we trained the classifier on $n-1$ datasets and computed the prediction accuracy on the $n^{\text {th }}$ dataset ${ }^{5}$. For low and medium traffic, prediction accuracy was reasonable high with means of $85.33 \%$ $(S D=11 \%)$ and $88 \%(S D=12 \%)$, respectively. For high traffic, however, prediction accuracy dropped to $67.33 \%(S D=18.73 \%)$. In addition to crossvalidation, random forests can do out-of-bag predictions by, at each bootstrap sample, predicting the data that is not in the sample using the tree grown with the particular bootstrap sample. Aggregating over these out-of-bag predictions, one can calculate an out-of-bag estimate of the classification error rate (Liaw \& Wiener, 2002, p. 18). For low, medium, and high traffic, these estimates are $84.62 \%, 87.70 \%$, and $64.06 \%$, respectively, mirroring the results of the crossvalidation routine. Note that the chance prediction accuracy is $1 / 6=.167$.

\footnotetext{
${ }^{5}$ Note that there are $n=50$ datasets for each driver profile. When I say "one dataset", I mean "one dataset for each driver".
} 


\section{Lane Changes in High Traffic}

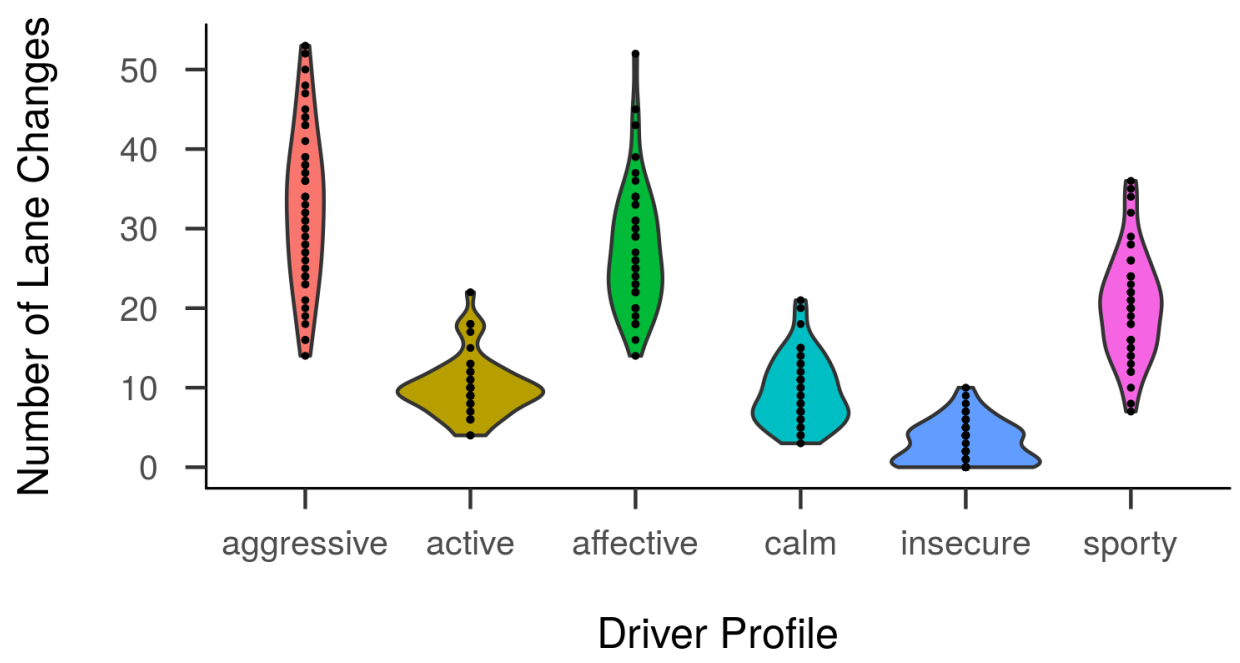

FIGURE 2.8: Shows violin plots for the number of lane changes for each dataset and each driver profile in high traffic.

What predictors do most of the work? Figure 2.9, 2.10, and 2.11 show the importance of the predictor variables for light, medium, and high traffic, respectively. In low and medium traffic settings, speed and headway seem to be the most crucial features; for both, the maximum speed - speed 100 - is the strongest predictor. For high traffic, headway is the best feature to discriminate between the driver profiles. Interestingly, the top predictor is the number of lane changes during the 75.9 kilometres of driving; Figure 2.8 shows violin plots for the number of lane changes for each dataset and driver profile. Based on this, one could easily discriminate aggressive, active, and sporty drivers from active, calm, and insecure ones, respectively. We can illustrate this by using multidimensional scaling to visualize the similarities between driver profiles.

\subsubsection{Clustering}

Figure 2.12 visualizes the similarity between the driver profiles for the different traffic scenarios. In low and medium traffic, the driver profiles can be well separated. The insecure driver is the most isolated one, while there exists a high overlap for aggressive and sporty 


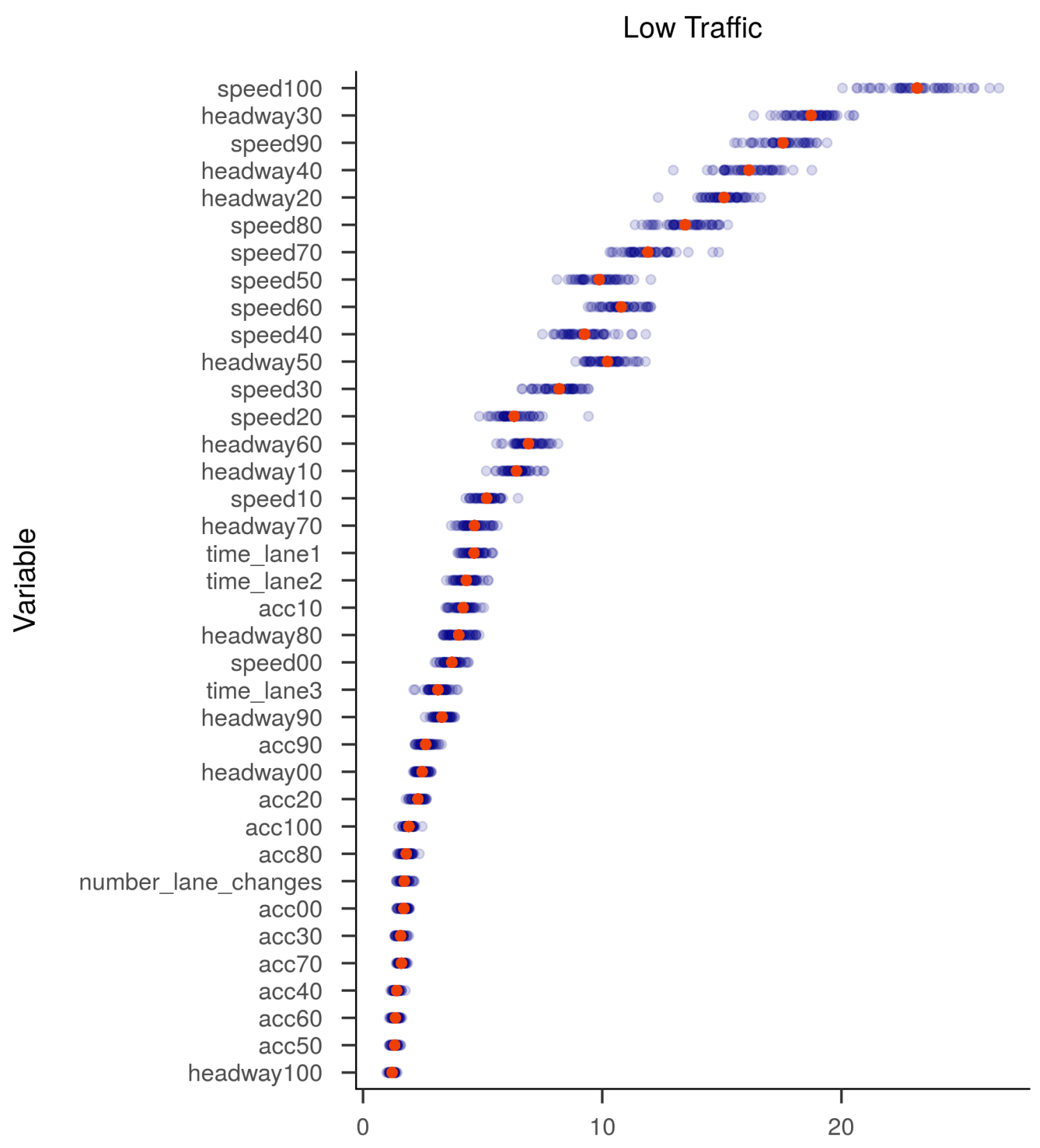

Gini Importance

FIGURE 2.9: Shows the mean decrease in Gini impurity for all variables for each of the steps in leave-one-out cross-validation on the light traffic simulation data. Red dots indicate the mean decrease for the fifty cross-validation steps. The number behind the variable name indicates the particular decile of the histogram. 


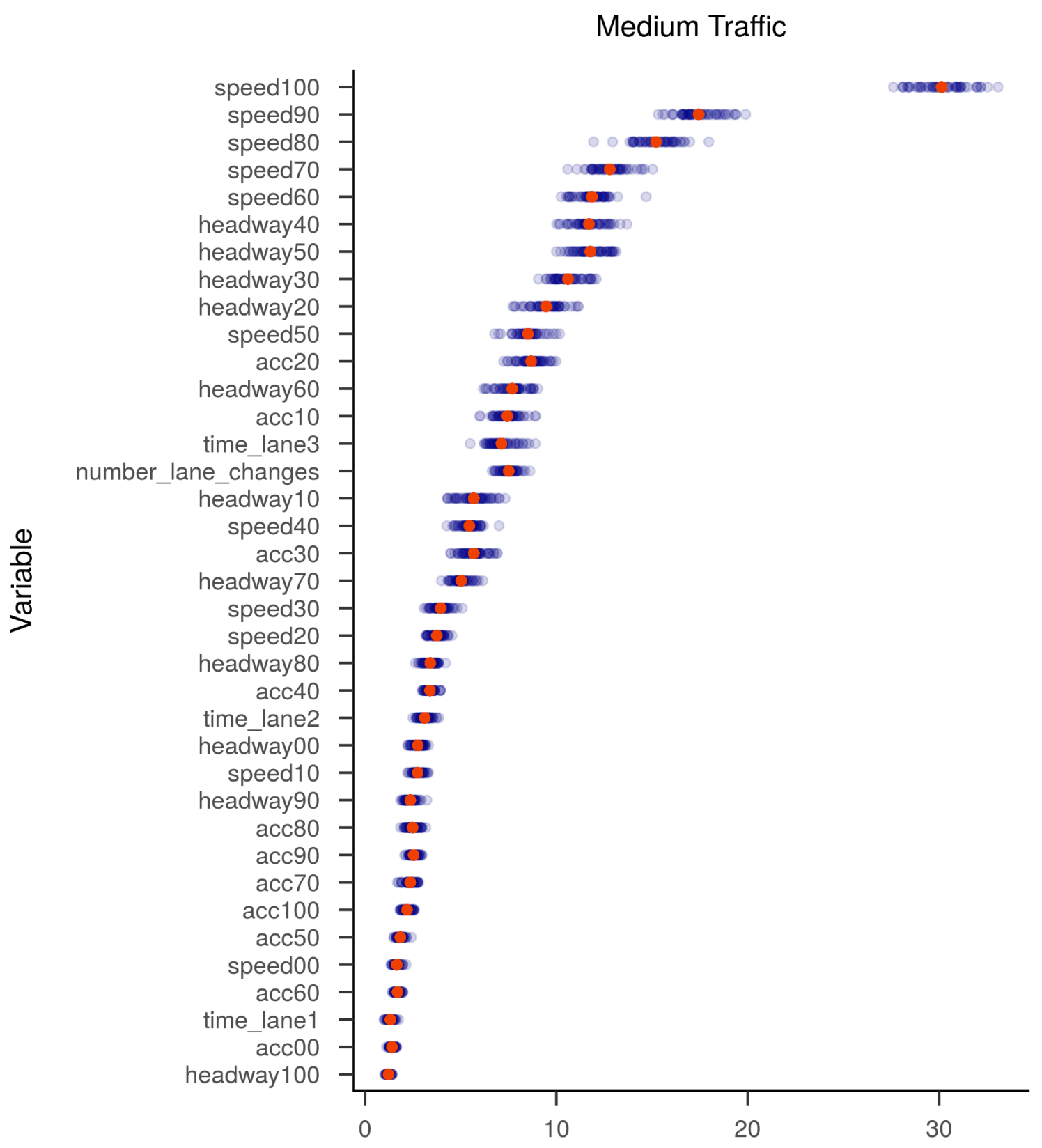

Gini Importance

FIGURE 2.10: Shows the mean decrease in Gini impurity for all variables for each of the steps in leave-one-out cross-validation on the medium traffic simulation data. Red dots indicate the mean decrease for the fifty cross-validation steps. The number behind the variable name indicates the particular decile of the histogram. 


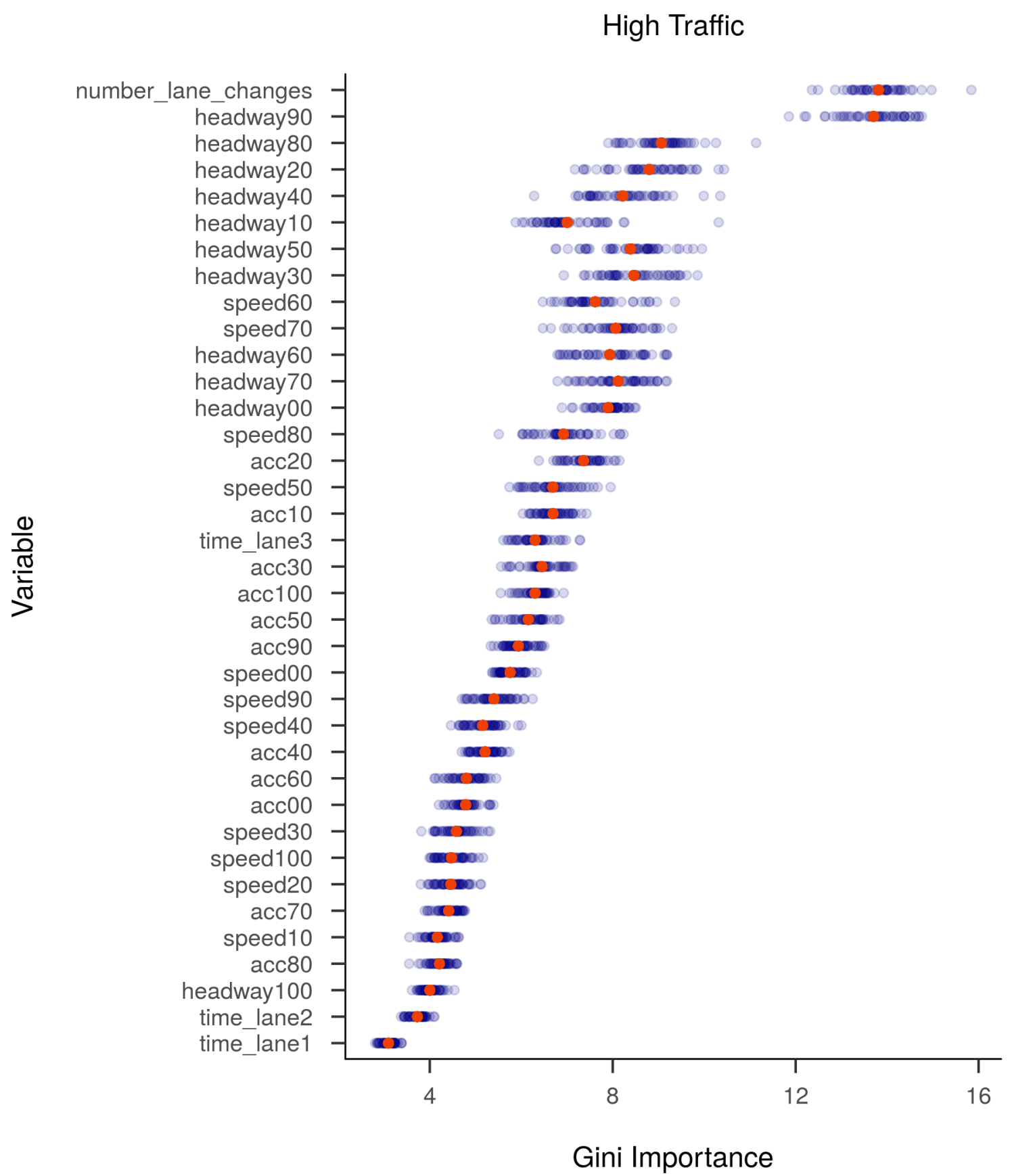

FIGURE 2.11: Shows the mean decrease in Gini impurity for all variables for each of the steps in leave-one-out cross-validation on the high traffic simulation data. Red dots indicate the mean decrease for the fifty cross-validation steps. The number behind the variable name indicates the particular decile of the histogram. 


\section{Low Traffic}

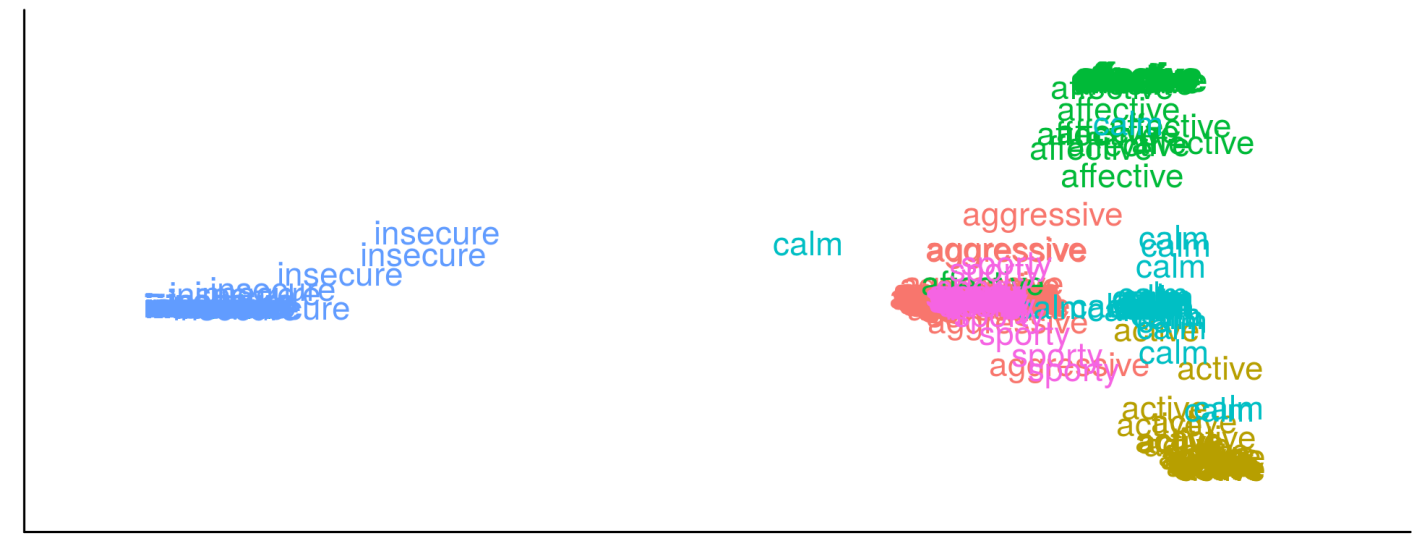

Medium Traffic

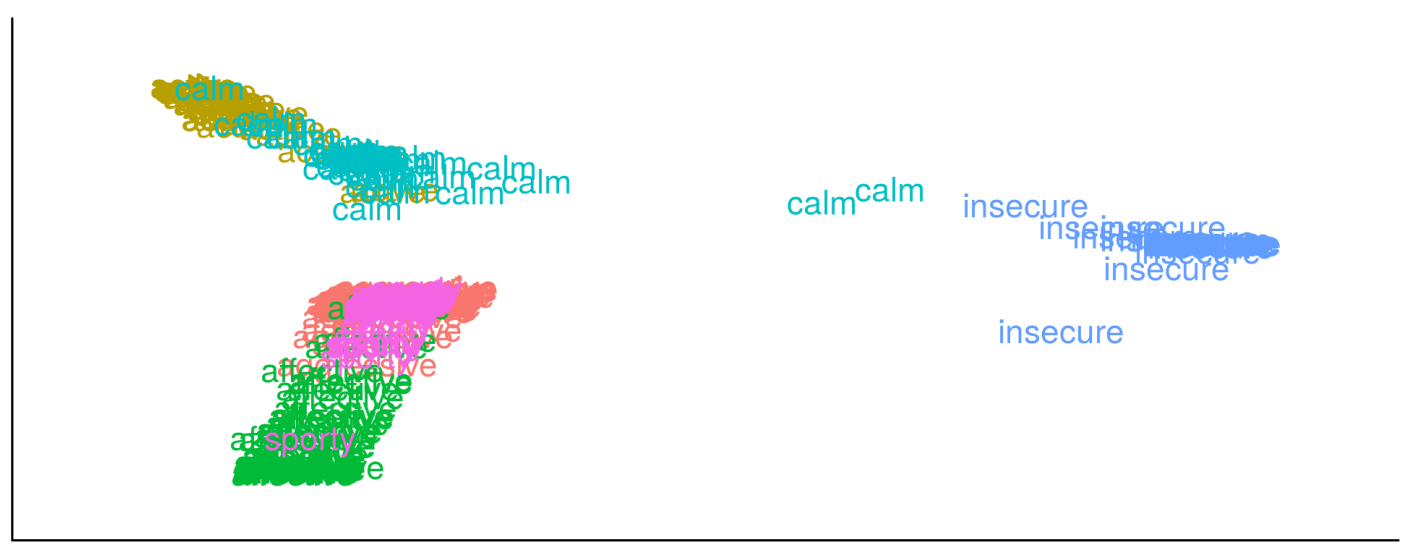

High Traffic

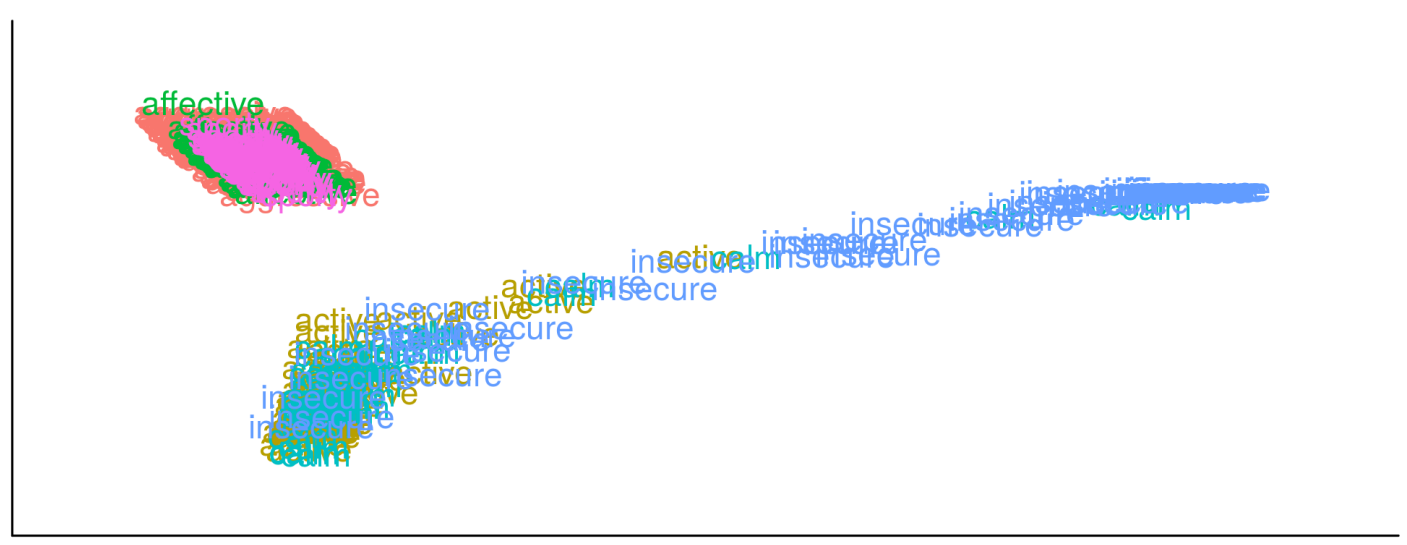

FIGURE 2.12: Shows the similarity between the driver profiles based on a multidimensional scaling analysis of the proximity matrix returned by random forests. From top to bottom, shows the clustering for low traffic, medium, and high traffic scenarios, respectively. 
drivers for both scenarios. In the high traffic scenario, affective, sporty, and aggressive drivers, as well as active, calm, and insecure drivers are difficult to seperate from each other, respectively.

\subsection{Discussion}

An important step in model validation is to check whether one can recover the parameters of the model when simulating from it (e.g., Heathcote, Brown, \& Wagenmakers, 2015, p. 29-31). Instead of parameters, we tested whether we can recover the assumed to be distinct driver profiles of the Daimler traffic simulation. We generated synthetic driving data via the agent-generating process described by the Daimler traffic simulation, and used random forests to recover the driver profiles. Additionally, we visualized the similarity between the profiles to discover potential overlaps.

Overall, our endeavour was successful: we achieved high prediction accuracy in both low and medium traffic scenarios, isolating all but the aggressive and sporty drivers, who overlapped substantially. In both scenarios, speed and headway were the strongest discriminatory features. Incidentally, sporty and aggressive drivers are highly similar on those two features (see Table 2.4); in fact, after collapsing those drivers, predictive accuracy increased to above $96 \%$ for both low and medium traffic scenarios. Separation of drivers was much worse in high traffic, with two broad clusters emerging: aggressive, sporty, affective drivers, and calm, insecure, active drivers. The feature with highest discriminatory power was the number of lane changes, which, as is shown in Figure 2.8, at most distinguishes between three related clusters. These results entail a number of practical implication for the Daimler traffic simulation, as well as for future studies. Below I discuss the limitations of the present study and outline its practical implications.

First, note that, as indicated in Table 2.4, aggressive and sporty drivers differ only in parameters concerning Consideration, that is, politeness and the bias to drive on the right lane. 
In the current version of the Daimler traffic simulation, all parameters within a category get assigned the same threshold, depending on the categorization of the main parameter. For example, aggressive and sporty drivers are both in group "-" for the category Safety, which means that the parameters in that category — DesiredHeadway and minGapFront — are drawn from the same tails of the empirically calibrated distribution, respectively. This means that they do not differ in speed and headway, the two features which most strongly discriminated between the other drivers. Practically, to increase separation between the two driver profiles, one might specify the parameters within a particular category differentially instead of treating them equally. For example, it strikes me as unintuitive that aggressive and sporty drivers should have the same desired headway, and one might increase the separation of the two classes by changing this and other parameters to make the differences between them more pronounced.

Second, in addition to specifying the parameters in the categories differentially, I recommend introducing a parameter that models driving behaviour across time: for example, an anger parameter that increases - like a boiler — in heat with situations that are annoying to the driver such as traffic or slower vehicles on the leftmost lane. After a number of such situations, the boiler might overheat which correspondingly decreases Consideration parameters (e.g., politeness). I suspect that setting such a parameter for certain driver profiles, say the aggressive one, will increase discriminability between the driver profiles, but also make the traffic simulation more realistic as a whole. Modeling approaches by other researchers already incorporate internal features of the driver, such as emotions (e.g., Cristea et al., 2016). Additionally, cognitive parameters such as reaction time or distraction could be included as well. However, while this would make the simulation more dynamic, such behaviour is difficult to calibrate because empirical data is hard to obtain for these behaviours. This makes benchmarks of recoverability, demonstrated in this research, all the more important.

The computer-generated agents are most frequently employed in driving simulator studies in which traffic situations are controlled and brief. Very rarely, if ever, do participants engage 
in free driving with traffic around them. This leads me to the next point.

Third, in the current study, each dataset consisted of 75.9 kilometres of driving. This was a rather arbitrary choice; could the driver profiles still be recovered when using, say, only half the amount of driving data? Or a third? In other words: what is the time scale of the system? How long do we need to observe the drivers to reliably distinguish between them? In driving simulator studies, in which this traffic simulation will be chiefly employed, participants usually observe drivers only for a short time, at certain critical situations such as overtaking or ascending onto a highway. Future simulation-based validation studies could construct such critical situations, and test whether the driver profiles can be distinguished.

The point above is related to the difference between low and high traffic scenarios, because the discriminability will be determined by the informativity of the situations. In high traffic scenarios, the drivers cannot reach their maximum desired velocity, which reduces the information we can draw from the observed data. This explains why discrimination was much lower in high traffic settings.

Fourth, while we could recover most of the driver profiles in low and medium traffic settings, it is not clear from the results that they resemble human driving behaviour; the results here are solely concerned with internal consistency: they were not compared to external benchmarks. Skeptically speaking, it can be conjectured that they do not, in fact, resemble human driving behaviour more broadly. First, while the use of empirical data on driving behaviour distinguishes the Daimler traffic simulation and avoids arbitrariness in calibrating parameter values, the data is based solely on Mercedes-Benz drivers and, therefore, might not reflect the general population. On the one hand, it might obscure the empirical distribution of the driver profiles; it is possible that, among Mercedes-Benz drivers, there are less insecure and calm drivers but more sporty and aggressive ones. On the other hand, the increased homogeneity might blur the distinctions on certain parameter values. Second, it is quite possible that the empirical foundation for six distinct driver profiles is not as strong 
as initially believed. The empirical basis for the driver profiles are a series of studies conducted between 1972 and 1993 (see Table 2.2). However, each of the studies had their own purpose. Moreover, the statistical analysis by Hürlimann (1996, p. 79-106) is questionable. First — as far as I could make out based on the short description in Hürlimann (1996) — the driver profiles are only based on the "MIKRO" study and have been uncritically employed in subsequent studies. Second, Hürlimann (1996) do not employ inferential statistics. Instead, they interpret arguably small differences in the data at face value. It is thus not clear whether the driver profiles are actually distinguishable based on features like speed or sensitivity to dangerous situations. In fact, the descriptive data often looks strikingly similar across some of the driver profiles. For example, in "MIKRO", 1042 motorists have been asked to rate the danger of seven traffic situations on a five point scale. The calm and active profile averaged 2.2 and 2.1, respectively, while the sporty and aggressive driver averaged 1.5 and 1.1, respectively. The affective and insecure driver averaged 3.8 and 4.7, respectively. If anything, only three or four groups can be distinguished based on these data. Regardless, it seems to me that the idea of six distinct driver profiles has pragmatic value: it does lead to more variability among computer-generated drivers, and, based on common sense reasoning, this variability seems sound. Our results here showed that this theoretical variability is found in the software implementation of the Daimler traffic simulation, constituting an important step towards its validation. We have discussed a number of limitations, and have provided practical recommendations towards improving and extending the traffic simulation. 


\section{Chapter 3}

\section{Human-based Validation}

\subsection{Validity of Driving Simulations}

Driving simulators have a variety of advantages compared to conducting studies on the real road (De Winter et al., 2012), but these only hold when driving simulators yield valid measurements. Validity refers to the extent to which behaviour elicited in the simulator generalizes to behaviour on real roads (e.g., Klüver et al., 2016). Note that, due to their complexity, driving simulators can never be validated in total. Instead, individual configurations, simulations, and manoeuvres must be validated piece by piece.

Two types of validity are discussed in the broader literature (Klüver et al., 2016, p. 79-89). Physical validity refers to the extent to which the simulation reflects the real environment, and is closely related to the concept of immersion. Behavioural validity, on the other hand, refers to the extent to which the behaviour of humans in the simulation corresponds to actual driving behaviour. Two specific forms of behavioural validity are relative and absolute validity. Relative validity means that participants do not exhibit different behaviour in the simulator compared to the real world, while absolute validity additionally entails that behaviour shown in the simulator does not differ from actual driving behaviour.

In the current research we focus on the behavioural validity of the computer-generated drivers. Particularly, we ask whether the novel aggressive and calm driver profiles of the Daimler traffic simulation improve upon an older version of drivers that did not have distinct driver profiles. 


\subsection{A Note on Simulator Sickness}

Simulator sickness threatens to diminish the validity of driving simulators as a measurement tool. Its symptoms include sweating, eyestrain, dizziness, stomach awareness, and even nausea (Kennedy, Lane, Berbaum, \& Lilienthal, 1993) which all have a detrimental influence on driving, invalidating the generality of inferences drawn in simulator studies. About five to ten percent of participants have to abort the experiment due to simulator sickness (Preuß, personal communication), but some participants might still proceed, disregarding or downplaying their symptoms. When participants proceed with the experiment despite experiencing simulator sickness, bias might be introduced. For example, Helland et al. (2016) found that simulator sickness caused participants to drive more carefully which resulted in lower average speed and fewer steering wheel reversals. While there are many theories on simulator sickness (see, e.g., Brooks et al., 2010, p. 789), the most prominent is the sensory conflict theory which argues that simulator sickness arises when visual, vestibular, and somatosensory information are in conflict with each other and the sensory experience deviates from one's own expectation (Reason \& Brand, 1975). However, it is still not clear what exactly causes the occurence of simulator sickness (for an overview, see Hale, Stanney, Keshavarz, Hecht, \& Lawson, 2014), but driving simulator characteristics seem to play a minor role (Klüver, Herrigel, Preuß, Schöner, \& Hecht, 2012).

In this study, we monitored simulator sickness throughout the experiment using the Fast Motion Sickness questionnaire (FMS; Keshavarz \& Hecht, 2011), which requires the participant to indicate on a scale from 1 to 20 how well she is. We aborted the experiment when the participant indicated a value greater than ten, which only occurred once. We did not include this participant in the subsequent analysis. Other participants might still have experiences slight simulator sickness, and we therefore included it as a control variable, together with social desirability and others, in the statistical models. 


\subsection{Goal of this Research}

In the present research, our goal is to take first steps in validating the traffic simulation by the Daimler simulation team which features six distinct driver profiles that are based on empirical studies conducted in the 1970s, 1980s, and 1990s (Hürlimann, 1996, p. 79-106). The questions we seek to answer are

- Is the behaviour of the aggressive and calm profiles perceived as being closer to human driving, respectively, than the behaviour of the old driver profiles?

- Is the behaviour of the aggressive and calm profiles perceived as being more realistic, respectively, than the behaviour of the old driver profile?

Our hypotheses are directed: we expect a positive effect of driver profile. In order to test these hypotheses, we use Bayesian hierarchical regression methods; but more on that below.

\subsection{Method}

All materials accompanying this article, including raw data and analysis code, can be found online at https://osf.io/dw5hr/?view_only=992545a0d49a4a0198a215388ee95e5f.

\subsubsection{Participants}

We utilized the driving simulator at the FKFS in Vaihingen, Stuttgart — Europe's largest eight-axis motion simulator (Baumann, Riemer, Liedecke, Rumbolz, \& Schmidt, 2012). We aimed at having 30 participants for our final analysis ${ }^{1}$. Due to technical issues, we tested 39 participants, using data from 32 of them for the quantitative analysis, and 36 for qualitative analysis. Data collection occurred between the $27^{\text {th }}$ of February and the $10^{\text {th }}$ of March, and

\footnotetext{
${ }^{1}$ This number is based on a contract that was concluded before my arrival.
} 
recruitment of participants was outsourced to Consilio $\mathrm{GmBH}$, and participant criteria were applied such that they mirror the age and sex of the driving population (Follmer et al., 2010); see Table 3.1.

TABLE 3.1: Age and sex of participants $(N=32)$.

\begin{tabular}{lccccc}
\hline & \multicolumn{5}{c}{ Age } \\
& $20-30$ & $31-40$ & $41-50$ & $51-60$ & $n$ \\
\hline Female & 3 & 5 & 3 & 4 & 15 \\
Male & 3 & 5 & 4 & 5 & 17 \\
\hline
\end{tabular}

\subsubsection{Procedure}

For each participant, one and a half hours were allocated. Upon arrival, each participant first received a safety instruction, and subsequently filled out a basic demographic questionnaire, the Fast Motion Sickness questionnaire (FMS; Keshavarz \& Hecht, 2011), and the Simulator Sickness Questionnaire (SSQ; Kennedy et al., 1993).

After an instruction (see supplementary materials online), participants were situated in the driving simulator, and were provided with about three minutes of free driving to get accustomed to the environment, leaving twenty-five minutes for experimental interventions. The simulation consisted of only highway driving. Participants were instructed that there are two types of drivers: those mirrored on real data, and those that are purely simulated ${ }^{2}$.

The experiment had two parts. In the first part, participants experienced four scenarios in which they had to judge both whether the driver was human or a computer, the realism of the driver's behaviour on a scale from 1 (unrealistic) to 7 (realistic), and how often they think this type of driving behaviour occurs in the real world. Participants were asked to provide reasons for all their answers.

\footnotetext{
${ }^{2}$ Note that while the new driver profiles are inspired and modeled on real driving behaviour, they do not constitute a one to one mapping with real driving behaviour.
} 
FIGURE 3.1: Overview of the driving simulator study.

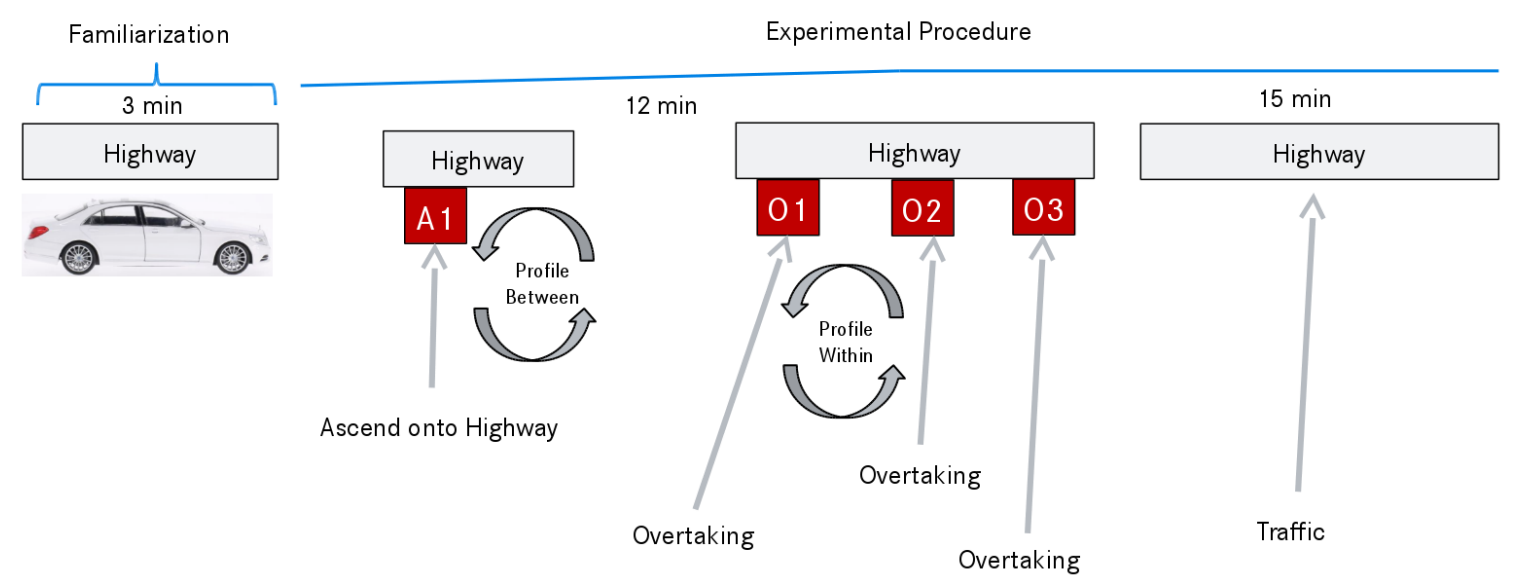

The first scenario consisted in ascending onto the highway; the other three consisted of being overtaken by other drivers. The first scenario had either the old, the aggressive, or the calm parameterization as a between factor; the last three scenarios had the parameterization as a within factor which were counterbalanced across participants. In this first phase, participants had to obey a speed limit of $100 \mathrm{~km} / \mathrm{h}$. Throughout the drive, an automated voice instructed participants and asked them questions related to the scenarios, including the FMS. After this first, controlled experimental part, participants engaged in fifteen minutes of free highway driving, obeying a speed limit of $120 \mathrm{~km} / \mathrm{h}$. In this phase, participants were surrounded with traffic and were asked to remark on anything that "seems off", unreal or unnatural. We included this part to discover potential "bugs" and create a list of desirable features to further increase the quality of the traffic simulation. For an overview of the whole procedure, see Figure 3.1.

After the experiment, participants were debriefed and asked to answer the FMS, questions about whether they have changed their driving style in the simulator compared to the real road, questions regarding their previous experience with driving simulators, a slightly modified version of the Slater-Usoh-Steed Presence Questionnaire(SUS; Slater, Usoh, \& Steed, 1995) to fit the driving context, and the Social Desirability Scale "Kurzskala Soziale Erwünschtheit-Gamma” (Kemper, Beierlein, Bensch, Kovaleva, \& Rammstedt, 2012). 
Overall, while the experimental side of this design included only aggressive and calm driver profiles, the exploratory part — in which all six driver profiles appeared — provided us with an impression of what participants think of the traffic simulation as a whole. While the first part was analyzed using quantitative methods, the second part required qualitative data analysis.

\subsubsection{Hypotheses and Statistical Rationale}

We expected an increase in judging the behaviour of other drivers as human and as realistic for aggressive and calm profiles, respectively, compared to the old parameterization. This means that our hypotheses are confirmatory and directed. For judgements on whether the driver behaves like a human or like a computer, we employed Bayesian hierarchical logistic regression; for the realism judgments, we employed Bayesian hierarchical ordered logistic regression. Thus, we conducted four tests, one for each dependent variable and driver profile, by dummy coding the predictor variable profile to be either 0 (old version of the traffic simulation) or 1 (aggressive or calm drivers, respectively), and computing Bayes factors (Kass \& Raftery, 1995). This approach raises the following questions: Why hierarchical models? Why logistic regression? Why ordered logistic regression? Why Bayesian inference? After briefly tackling each of these, I sketch the specification of the two Bayesian models and motivate the choice of priors.

\section{Why Hierarchical Models?}

Historically, there have been two ways of modeling data from psychological experiments: either ignoring variation among participants and using a single model for the averaged data, or using a model for each individual subject. The latter acknowledges the potential bias introduced by averaging, but suffers from lower efficiency, that is, less precise estimates because it uses data only from a single participant. Recently, mixed effects or multilevel models 
have been introduced to cognitive science which elegantly navigate this tradeoff by including both terms that are allowed to vary across participants and terms that are constant across participants (Baayen, Davidson, \& Bates, 2008; Sorensen \& Vasishth, 2015). Those models also lead to more accurate predictions because they pool information across participants and thus avoid overfitting (Efron \& Morris, 1977; Gelman, 2006).

However, there is considerable debate in the literature as to how to specify the varying or "random" compared to the non-varying or "fixed" model part (Barr, Levy, Scheepers, \& Tily, 2013; Bates, Kliegl, Vasishth, \& Baayen, 2015; Matuschek, Kliegl, Vasishth, Baayen, $\&$ Bates, 2017). Below, we allow for variation only in the intercept ${ }^{3}$; the effect of the predictors is assumed to be the same across participants. This means, in other words, that we allow participants to differ in their tendency to judge the behaviour of a computer-generated agent as being human and realistic, but we assume that the aggressive and calm driver profiles, respectively, exert the same effect on those judgements across participants. We think that this restriction does make sense mostly for practical reasons: adding varying slopes would add two more parameters, one describing the variation in the effect, another describing the correlation between varying intercepts and slopes. Given the small sample size, such a model strikes us as overparameterized ${ }^{4}$.

\section{Why Logistic Regression?}

General linear models, which include the $t$-test, the analysis of variance, and multiple linear regression (Cohen, 1968), model the (continuous) response variable as sum of a linear combination of predictor variables and zero-centered, normally distributed errors with constant variance (for an overview, see Poline \& Brett, 2012). However, the dependent variable we are analysing — whether the driver was judged as being a human or a computer — is binary,

\footnotetext{
${ }^{3}$ This is also necessary for generalizing the results beyond the sample to the broader population.

${ }^{4}$ However, although guided by simplicity, this is not a particularly good argument: it is an empirical question whether we can draw sensible conclusions from such models. In an online appendix, we estimate those models and report our findings. Note that none of the conclusions based on the varying intercepts only models change.
} 
which means that the assumption of a constant error variance is violated (see Jaeger, 2008, p. 436). Jaeger (2008) finds that there is wide-spread use of applying analysis of variance techniques to the averaged data, that is, proportions, which can lead to impossible predictions (proportions larger than one) and spurious interaction effects. Logistic regression models the dependent variable on its proper domain (more on that below), and thus is preferable to the general linear model for categorical data.

\section{Why Ordered Logistic Regression?}

The arguments for ordered logistic regression instead of linear regression are similar as above. First, realism judgements are not on the real line, but are restricted to categories from 1 to 7. Thus, applying linear regression could again yield impossible predictions. Moreover, by assuming a continuous response variable, linear regression would imply an interval scaled response. An interval scale would mean that the difference between 1 and 2 were the same as the difference between 5 and 6 (Stevens, 1946), an assumption too strong for our level of measurement. Beyond this theoretical argument, the realism judgements, conditional on the predictor, are highly skewed (see Figure 3.4), violating the assumption that the response variable is (conditionally) normally distributed. Therefore, we model the response variable on its proper domain, merely assuming that realism judgement are ordered, such that, for example, a judgement of 6 indicates that the driving behaviour was judged as being more realistic than a judgement of 2 would indicate. Ordered logistic regression allows us to encode this information by modeling the log cumulative odds (more on that below).

\section{Why Bayesian inference?}

Throughout the history of statistics, and the 20th century in particular, debates about the respective merits of classical or frequentist and Bayesian statistics abounded (McGrayne, 
2011). I do not want to add to these debates, but only briefly justify my choice ${ }^{5}$.

Null hypothesis testing via $p$-values has been, and still is, the dominant approach in the social and medical sciences even though this practice has received scathing critique throughout the decades (e.g., Berkson, 1938; Wagenmakers, 2007), and is misapplied and misunderstood by a majority of researchers, practitioners, and even statisticians (e.g., Oakes, 1986; Haller \& Krauss, 2002; Hoekstra, Morey, Rouder, \& Wagenmakers, 2014; Wulff, Andersen, Brandenhoff, \& Guttler, 1987; Lecoutre, Poitevineau, \& Lecoutre, 2003). Practically, I do not think $p$-values provide an appropriate answer to the question: do aggressive and calm driver profiles improve upon older versions of the drivers? This is because the $p$-value is defined as the probability of observing a summary statistic of the data at least as extreme as the one we have observed, assuming that the null hypothesis is true. Conventionally, if $p<.05$, we reject the null hypothesis in favour of an alternative hypothesis; but we did not actually compare it to the alternative - we only looked at the data under the null hyothesis (cf., Rouder, Morey, Verhagen, Province, \& Wagenmakers, 2016). Thus, $p$-values merely check whether the null hypothesis conforms to the data, but are inappropriate for model comparison 6 .

In contrast, Bayesian statistics allows us to directly compare models by assessing how well they predict the data actually observed. Bayesian statistics derives from axiomatic probability theory and conceptualizes probability as degree of belief (Lindley, 2000). To estimate parameters of a model $\mathcal{M}$, we combine the likelihood and the prior over those parameters using Bayes' rule

$$
\underbrace{p(\theta \mid y, \mathcal{M})}_{\text {Posterior }}=\frac{\overbrace{p(y \mid \theta, \mathcal{M})}^{\text {Likelihood }} \overbrace{p(\theta \mid \mathcal{M})}^{\text {Prior }}}{\underbrace{p(y \mid \mathcal{M})}_{\text {Marginal Likelihood }}}=\frac{p(y \mid \theta, \mathcal{M}) p(\theta \mid \mathcal{M})}{\int_{\Theta} p(y \mid \theta, \mathcal{M}) p(\theta \mid \mathcal{M}) \mathrm{d} \theta} .
$$

\footnotetext{
${ }^{5}$ See the online appendix at https://osf.io/y6nmd/?view_only=992545a0d49a4a0198a215388ee95e5f for a more thorough treatment of the history and different philosophies of the respective inference paradigms.

${ }^{6}$ Again, the online appendix provides a more nuanced treatment.
} 
With the advent of Markov chain Monte Carlo methods and user-friendly software such as Stan (Carpenter et al., 2017), estimating the posterior distribution of parameters in complex models has become tractable. Those methods have found clever ways to avoid computing the marginal likelihood, a potentially high dimensional integral. However, convergence checks have to be conducted to make sure that the Markov chains have converged and one samples from the stationary distribution which is the posterior distribution (e.g., Jackman, 2009, ch. 4). We use the $\hat{R}$ statistic to assess convergence (Gelman \& Rubin, 1992).

We can apply Bayes' rule not only on parameters, but also on models. Stating it in terms of odds instead of probabilities, the posterior odds for two models $\mathcal{M}_{1}$ and $\mathcal{M}_{0}$ becomes

$$
\underbrace{\frac{p\left(\mathcal{M}_{1} \mid y\right)}{p\left(\mathcal{M}_{0} \mid y\right)}}_{\text {Posterior odds }}=\underbrace{\frac{p\left(y \mid \mathcal{M}_{1}\right)}{p\left(y \mid \mathcal{M}_{0}\right)}}_{\text {Bayes factor }} \times \underbrace{\frac{p\left(\mathcal{M}_{1}\right)}{p\left(\mathcal{M}_{0}\right)}}_{\text {Prior odds }} .
$$

The ratio of marginal likelihood, known as the Bayes factor (Kass \& Raftery, 1995), describes the shift in belief from prior odds to posterior odds that is brought about by the data; it thus provides a continuous measure of evidence (Morey, Romeijn, \& Rouder, 2016). Using odds instead of probabilities has the added benefit that we need not specify an exhaustive set of models, whose priors sum to unity, which would force us to believe the disjunction of the models with absolute certainty (cf. Morey, Romeijn, \& Rouder, 2013, p. 72). In fact, using odds means that we can be agnostic about the number of models under consideration and need not even accept that the true model is among them (Morey et al., 2013, p. 72).

Although computing the Bayes factor for any two arbitrary models is difficult because it entails computing marginal likelihoods, we can utilize a computational trick known as the Savage-Dickey density ratio because we are dealing with nested models, that is, when the null model is a special case of the alternative model (Wagenmakers, Lodewyckx, Kuriyal, \& Grasman, 2010). Using this trick, the Bayes factor in favour of the null model $\mathcal{M}_{0}$ compared 
to the alternative model $\mathcal{M}_{1}$ can be calculated as

$$
\mathrm{BF}_{01}=\frac{p\left(\beta=0 \mid y, \mathcal{M}_{1}\right)}{p\left(\beta=0 \mid \mathcal{M}_{1}\right)}
$$

where $\beta$ denotes the parameter of interest, the numerator denotes the density of the marginal posterior distribution of $\beta$ at point zero, and the denominator denotes the density of the marginal prior distribution of $\beta$ at point zero. Viewing the Bayes factor from this angle also makes apparent its sensitivity to the prior: wide, uninformative priors decrease the denominator and thus increases the Bayes factor in favour of the null model.

Our predictions are directed: we assume, for both calm and aggressive driver profiles, an increase both in the probability of judging the drivers as human and in the realism ratings. We map these theoretical hypotheses into statistical ones by computing directed Bayes factors. That is, we compare a model which assumes a positive effect of the driver profiles to the null model. Incorporating this prior knowledge increases the efficiency of our hypothesis testing because we make bolder predictions. If we are correct, we get a boost in credibility; however, if we are wrong and the effect is in the opposite direction, we lose credibility. Being bold in one's predictions is important for theory building more generally (e.g. Platt, 1964), and specifying the direction of an effect seems like a first step in the right direction.

We use results from Klugkist and Hoijtink (2007), which generalize the Savage-Dickey density ratio (Wetzels, Grasman, \& Wagenmakers, 2010), in order to compute the Bayes factor in favour of the full model, $\mathcal{M}_{1}$, compared to the restricted model which assumes a positive effect, $\mathcal{M}_{+}$. We can use this resulting Bayes factor to compute the Bayes factor we are actually interested in - the Bayes factor in favour of the order-restriction compared to the null model — by exploiting transitivity

$$
\begin{aligned}
\mathrm{BF}_{+0} & =1 / \mathrm{BF}_{1+} \times \mathrm{BF}_{10} \\
& =\frac{p\left(y \mid \mathcal{M}_{+}\right)}{p\left(y \mid \mathcal{M}_{1}\right)} \times \frac{p\left(y \mid \mathcal{M}_{1}\right)}{p\left(y \mid \mathcal{M}_{0}\right)}=\frac{p\left(y \mid \mathcal{M}_{+}\right)}{p\left(y \mid \mathcal{M}_{0}\right)} .
\end{aligned}
$$


TABLE 3.2: Evidence categories for the Bayes factor $B F_{10}$. From Table 4 in Vandekerckhove, Matzke, and Wagenmakers (2015).

\begin{tabular}{cccl}
\hline \multicolumn{2}{c}{ Bayes factor $B F_{10}$} & Interpretation \\
\hline & $>$ & 100 & Extreme evidence for $\mathcal{M}_{1}$ \\
30 & - & 100 & Very strong evidence for $\mathcal{M}_{1}$ \\
10 & - & 30 & Strong evidence for $\mathcal{M}_{1}$ \\
3 & - & 10 & Moderate evidence for $\mathcal{M}_{1}$ \\
1 & - & 3 & Anecdotal evidence for $\mathcal{M}_{1}$ \\
& 1 & & No evidence \\
$1 / 3$ & - & 1 & Anecdotal evidence for $\mathcal{M}_{0}$ \\
$1 / 10$ & - & $1 / 3$ & Moderate evidence for $\mathcal{M}_{0}$ \\
$1 / 30$ & - & $1 / 10$ & Strong evidence for $\mathcal{M}_{0}$ \\
$1 / 100$ & - & $1 / 30$ & Very strong evidence for $\mathcal{M}_{0}$ \\
& $<$ & $1 / 100$ & Extreme evidence for $\mathcal{M}_{0}$ \\
\hline
\end{tabular}

While Bayes factors constitute a continuous measure of evidence, it makes sense to create categories and attach verbal labels to them for communication purposes; see Table 3.2. For introductions to Bayesian statistics to psychology and cognitive science, see Etz, Gronau, Dablander, Edelsbrunner, and Baribault (2017), Sorensen and Vasishth (2015), McElreath (2016).

\subsubsection{Model Specification}

\section{Hierarchical Logistic Regression}

In this model, each data point $i$ of each participant $j, y_{i j}$, is assumed to come from a Bernoulli distribution with one participant-specific parameter, $\theta_{j}$, which denotes the probability of judging a driver as being human or a computer

$$
y_{i j} \sim \operatorname{Bernoulli}\left(\theta_{j}\right)
$$


$\theta_{j}$ is modelled via a real-valued, linear predictor $\eta_{j}$. In order to map $\eta_{j}$ onto the domain of the Bernoulli distribution, which is $[0,1]$, we use the logistic function (see also Figure 3.2)

$$
\theta_{j}=\frac{1}{1+e^{-\eta_{j}}}
$$

where $\eta_{j}=\left(X^{z} \cdot \beta\right)_{j}+\xi_{j}$ is a linear combination of the predictor variables. $X^{z}$ is the $n \times p$ standardized design matrix (which includes an intercept term) where $n$ is the number of observations and $p$ is the number of predictors, and $\beta$ is the $p \times 1$ vector of regression weights to be estimated. $\xi_{j}$ accounts for participant-specific differences in the intercept, rendering the model hierarchical.

In a Bayesian framework, priors are explicitly specified. This has three benefits. First, the subjective element in the analysis is made transparent and can be rigorously scrutinized (Berger \& Berry, 1988; Rouder, Morey, \& Wagenmakers, 2016); second, priors allow for the analysis to be extended by, well, prior knowledge, which is especially important in small sample studies because it makes statistical inference more efficient ${ }^{7}$; and third, sensible priors act as regularizer and avoid overfitting (McElreath, 2016, pp. 186).

Note that the Bayes factor is a ratio of marginal likelihoods and thus depends on the prior distribution over the parameters. This makes sense because the prior, together with the likelihood, constitutes the model which can make predictions about data (see also Vandekerckhove, Matzke, \& Wagenmakers, 2015). This explains why it does not make much sense to specify wide, uninformative priors, because they decrease the predictive power of the model, as encoded by the marginal likelihood; when using, for example, a uniform prior over the entire real line for some parameter, the marginal likelihood becomes zero for the model which includes this parameter compared to a model that does not include this parameter, a fact that is known as Lindley's paradox (Lindley, 1957; DeGroot, 1982). Thus, care must be

\footnotetext{
${ }^{7}$ Care must be taken not to use uninformative priors as they can actually hamper inference in small sample settings because the data fail to override the priors (McNeish, 2016).
} 
taken when specifying priors over parameters that are of interest from a model comparison standpoint.

Specifying such priors for generalized linear models is more complicated than for simple linear models because of the non-linear mapping occurring through the linking function. For a simple linear model such as the $t$-test, priors can be put on the intuitive effect size measure Cohen's $d$, which describes the standardized difference between two groups (Rouder, Speckman, Sun, Morey, \& Iverson, 2009). Zero-centered Cauchy priors have been suggested as default priors for these problems (Liang, Paulo, Molina, Clyde, \& Berger, 2008; Rouder \& Morey, 2012) due in part to their mathematical convenience.

For logistic regression, we have to respect the mapping which is achieved through a logistic function, which is plotted in Figure 3.2. Changes in the linear predictor, $X \beta$, lead to changes in the probability of judging a car as being driven by a human or computer, as denoted by $\theta$. For computing Bayes factors, we assume a Cauchy distribution with $r=1$ for the coefficient of the $0 / 1$ coded predictor which indicates whether the driver was from an older version of the traffic simulation (0), or a calm or aggressive driver (1), respectively. This prior holds $50 \%$ of its probability within the -1 and 1 bounds, which denote a decrease (for $\beta=-1$ ) or an increase (for $\beta=1$ ) of about $23.1 \%$ in the probability of judging a car as being driven by a human or a computer. On a population level, this would mean that there is an increase (or decrease) of $23.1 \%$ of judging cars as human for aggressive or calm drivers compared to the old parameterization, respectively. Figure 3.2 indicates these boundary points. Due to its fat-tailed nature, larger effect sizes are also possible. For the variables we want to adjust our inference for, such as simulator sickness, age, etc., we use independent, zerocentered Cauchy distributions with scale parameter $r=2.5$, which are weakly informative priors (Gelman, Jakulin, Pittau, \& Su, 2008). All in all, this prior specification strikes us as reasonable, but we will also report a sensitivity analysis which shows how the Bayes factor changes as a function of the Cauchy prior width over the parameter of interest.

The participant-specific offset $\xi_{j}$ from the average intercept $\beta_{0}$ is given zero-centered normal 
$\beta \sim \operatorname{Cauchy}(0,1)$

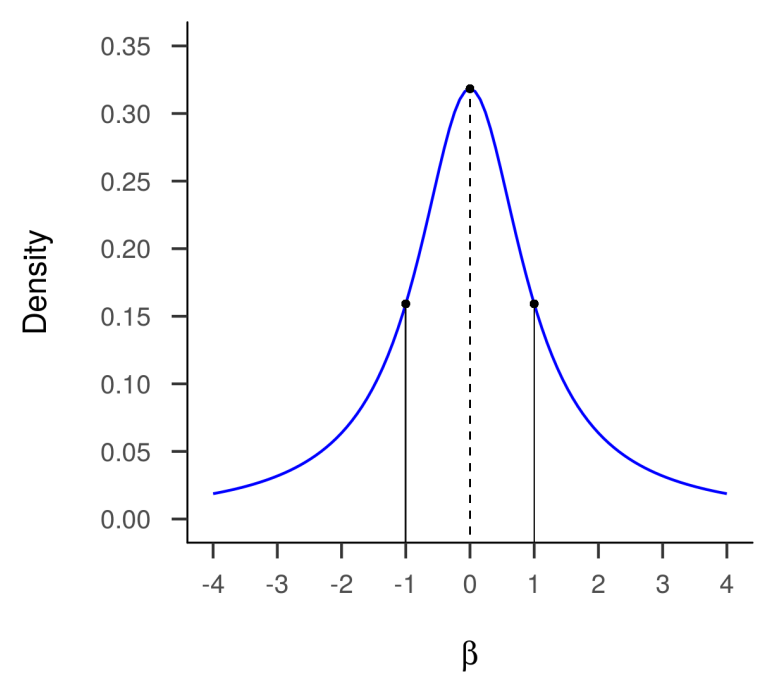

Sigmoid function

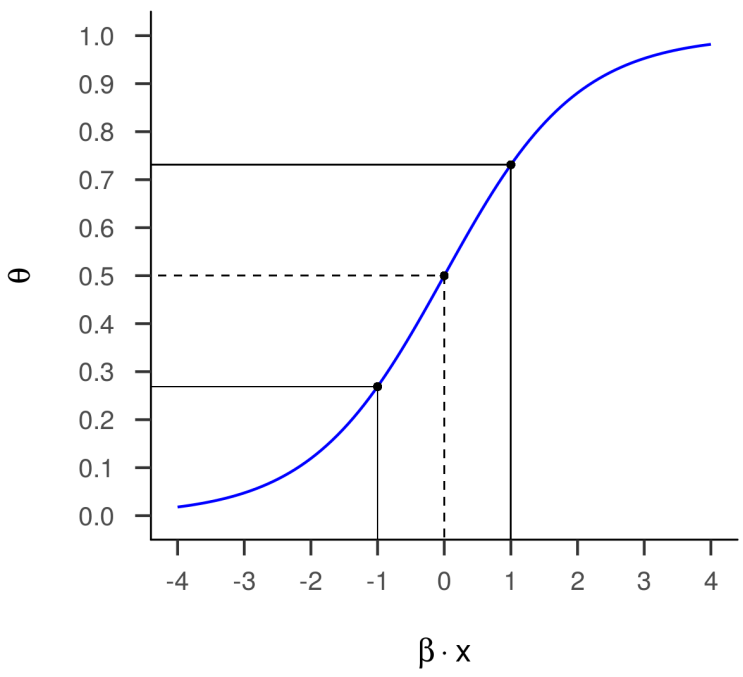

FIGURE 3.2: Shows how the Cauchy prior on $\beta$, which is the coefficient of the $0 / 1$ coded predictor $x$, influences the choice probability.

prior with standard deviation $\sigma$, which is given a zero-centered, half Student-t prior with ten degrees of freedom. Specifically,

$$
\begin{aligned}
\xi_{j} & \sim \operatorname{Norm}(0, \sigma) \\
\sigma & \sim \operatorname{Student-t}(10)_{I(0, \infty)}
\end{aligned}
$$

that is, $\xi_{j}$ models the participant-specific offsets from the grand mean which have a standard deviation $\sigma$.

\section{Hierarchical Ordered Logistic Regression}

Above we modeled choice probabilities, that is, the probability of a participant indicating that the driver was a human or a computer, by using a linear model in log odds space. Ordered logistic regression extends logistic regression by allowing the number of categories $K$ to be larger than two, as well as incorporating order information. To keep it consice, the interested reader is referred to McElreath (2016, ch. 11), for details on ordinal regression. 
Note simply that the prior specification here resembles the prior specification for logistic regression.

In summary, we used the R package brms (Bürkner, 2017) which utilizes the software program Stan (Carpenter et al., 2017) to fit the models, scaling all non-binary predictors by dividing by two times their standard deviation (Gelman, 2008) and using weakly informative Cauchy priors (Gelman et al., 2008).

\subsection{Results}

The raw as well as the tidy data (Wickham, 2014) and the analysis code are available from https://osf.io/dw5hr/?view_only=992545a0d49a4a0198a215388ee95e5f.

\subsubsection{Confirmatory Hypothesis Testing}

\section{Human/Computer Judgements}

The mean and standard deviation of the aggregated data are displayed in Figure 3.3, indicating that the data seem to confirm to our predictions. Computing directed Bayes factors as detailed above, we find that a model that assumes a positive effect of aggressive drivers is 22.43 times more likely than one that does not, that is, $\mathrm{BF}_{+0}^{\mathrm{aggr}}=22.43$, which indicates strong evidence, see Table 3.2. The results are equivocal for calm drivers, with a Bayes factor in favour of an effect of $\mathrm{BF}_{+0}^{\text {calm }}=1.15$, indicating a lack of evidence to prefer any particular model. These results are robust to the prior width specification, see Figure 3.5.

\section{Realism Judgements}




\section{Proportion judged Human}

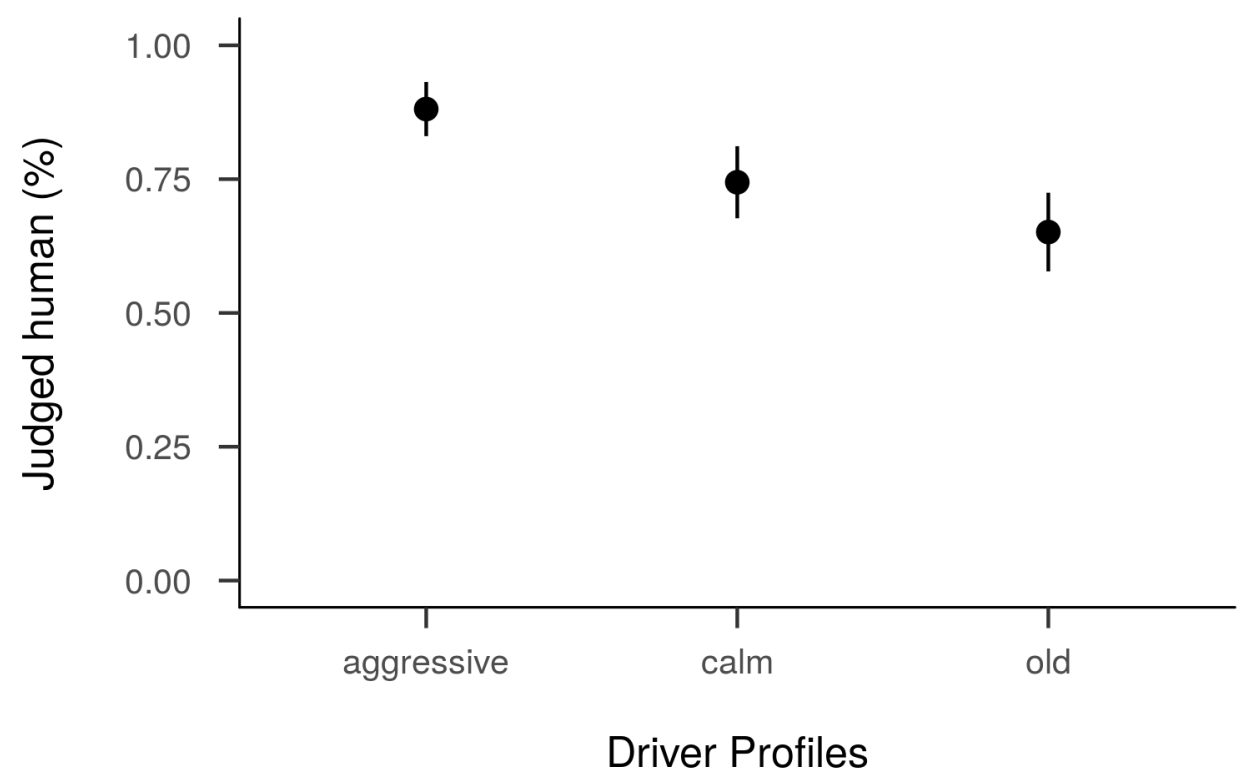

FIGURE 3.3: Shows mean and standard deviation of the proportion judged human for the respective driver profiles.

The realism judgements across all participants and driver profiles together with their median are displayed in Figure 3.4. The confirmatory hypothesis testing results were less pronounced compared to above. The models assuming that the aggressive and calm driver profiles are perceived as being more realistic compared to the old version are only $\mathrm{BF}_{+0}^{\text {aggr }}=2.03$ and $\mathrm{BF}_{+0}^{\mathrm{calm}}=1.17$ more likely than a model assuming no effect, respectively. This indicates that we have too little data to draw conclusions either way. These results are again robust to the prior width, see Figure $3.5^{8}$.

\subsubsection{Exploratory Analysis}

Through our Bayes factor analysis, we have confirmed our hypotheses only with respect to the aggressive driver being judged more frequently as being human. The other hypotheses

\footnotetext{
${ }^{8}$ Note that the Bayes factor in favour of the alternative model decreases with increasing prior width, which is as expected (see the Savage-Dickey density ratio above).
} 


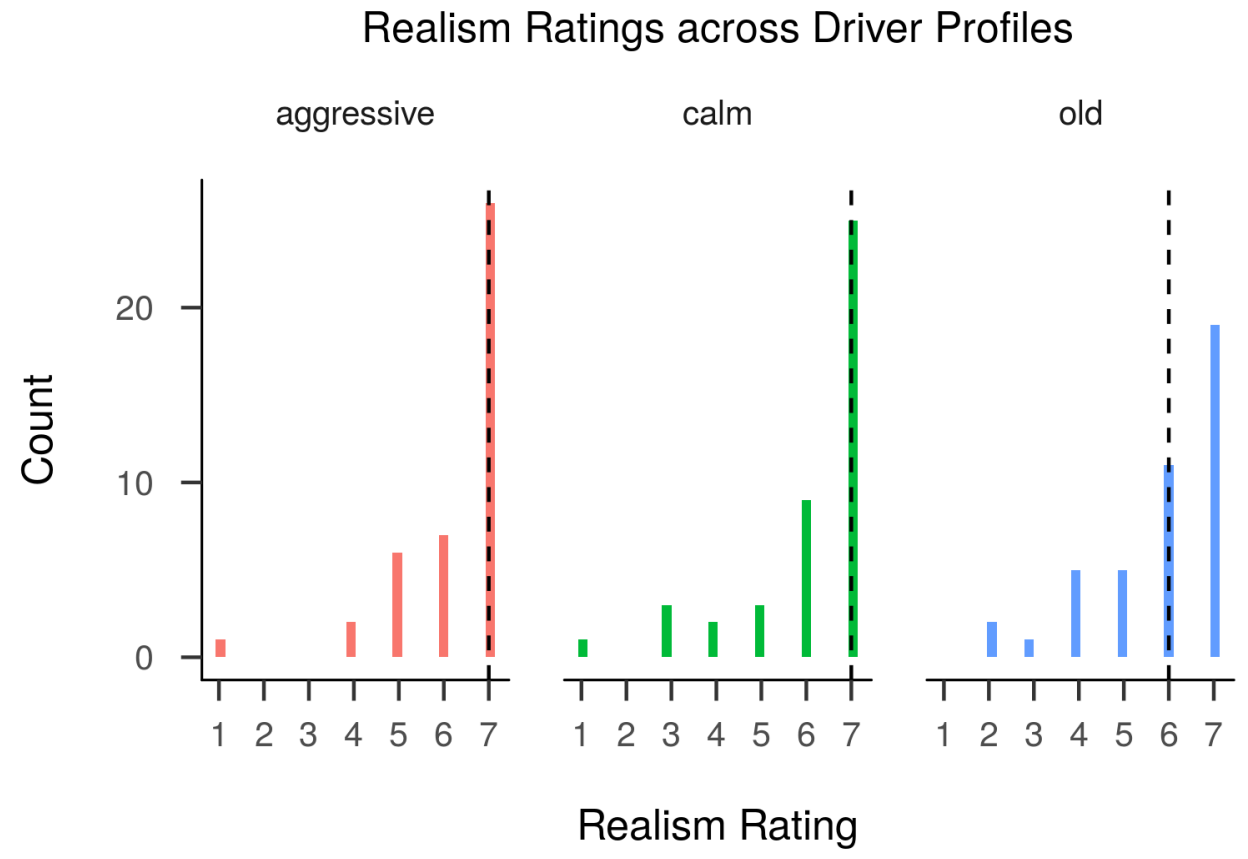

FIGURE 3.4: Displays realism ratings across driver profiles. Dashed black lines indicate the respective medians.

tests were equivocal, indicating the need to collect more data. We believe that it is illuminating, in addition to reporting confirmatory hypotheses tests, to also estimate a full Bayesian model and display the posterior distribution over all parameters (e.g., Kruschke \& Liddell, 2017) in order to see whether there is something else going on. Because we do not do any model comparison in this section, but only focus on parameter estimation, the choice of prior distribution becomes less crucial. Therefore, we have specified a Cauchy prior with a scale parameter of 2.5 - still a weakly informative prior to regularize our inferences - for all predictor variables; Figure 3.6 displays the means and the $95 \%$ credible intervals for all posterior distributions.

Except for the standard deviations, which are positive by definition, the marginal posterior distributions of predictor variables do not exclude zero. Interestingly, this is true for the dummy coded profile_aggr predictor variable in the logistic model, too. In this full model, we have included the variable scenario, which dummy codes whether the scenario was ascending onto the highway or being overtaken. Conditioning on this variable reveals different 


\section{Bayes factors for Human vs. Computer Judgements}

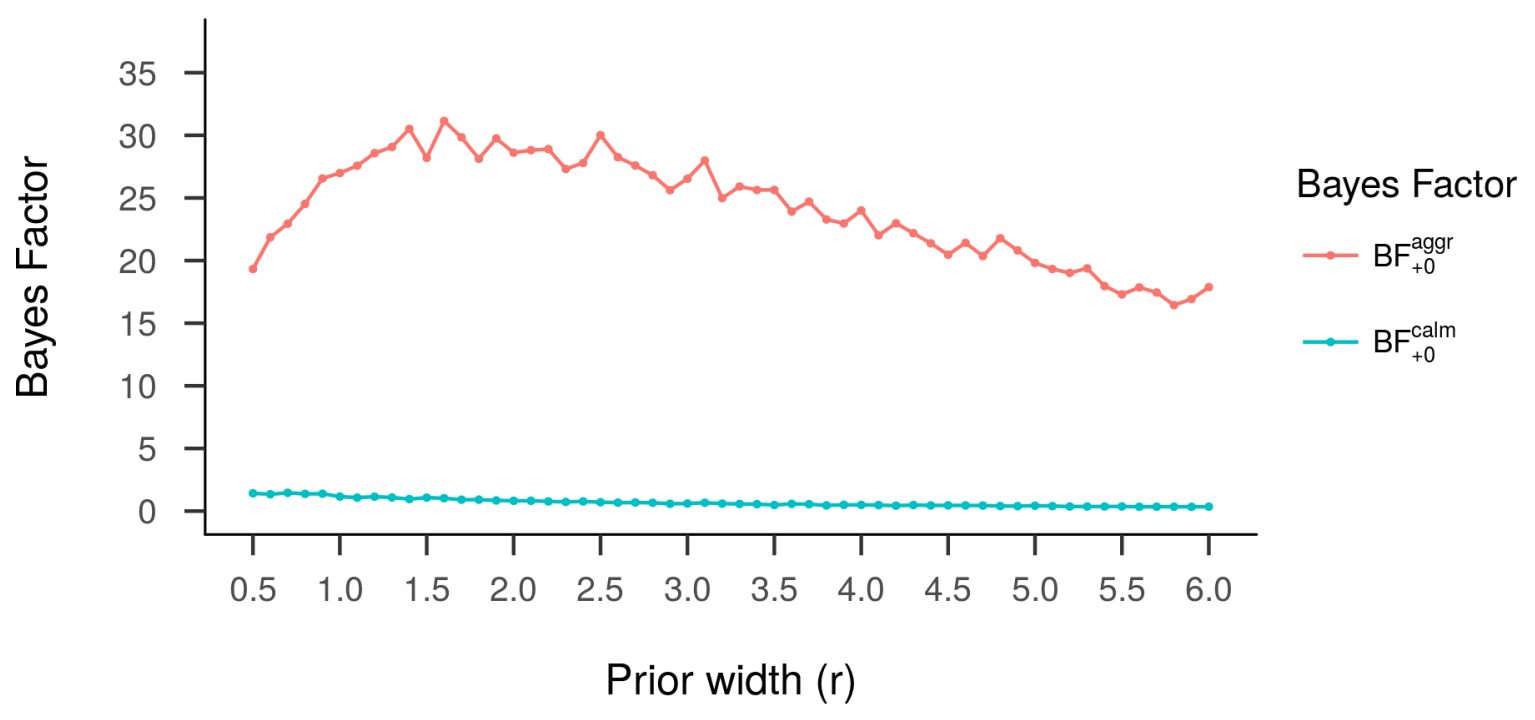

\section{Bayes factors for Realism Ratings}

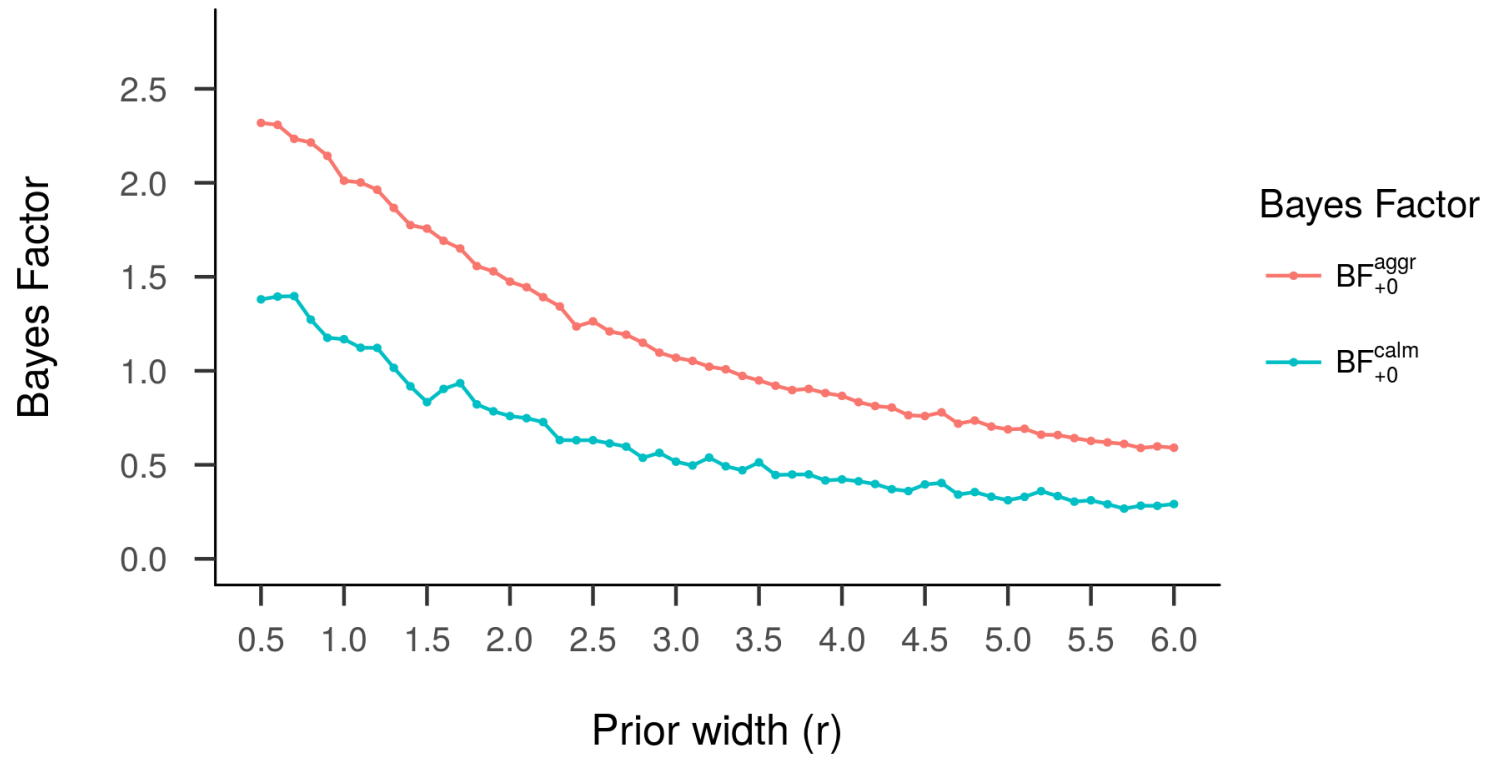

FIGURE 3.5: Shows the Bayes factors in favour of the directed hypotheses for different Cauchy prior widths. The higher the value of $r$, the greater the probability of a larger effect. The top plot displays the results for the logistic models, the bottom plot the results for the ordinal models. 


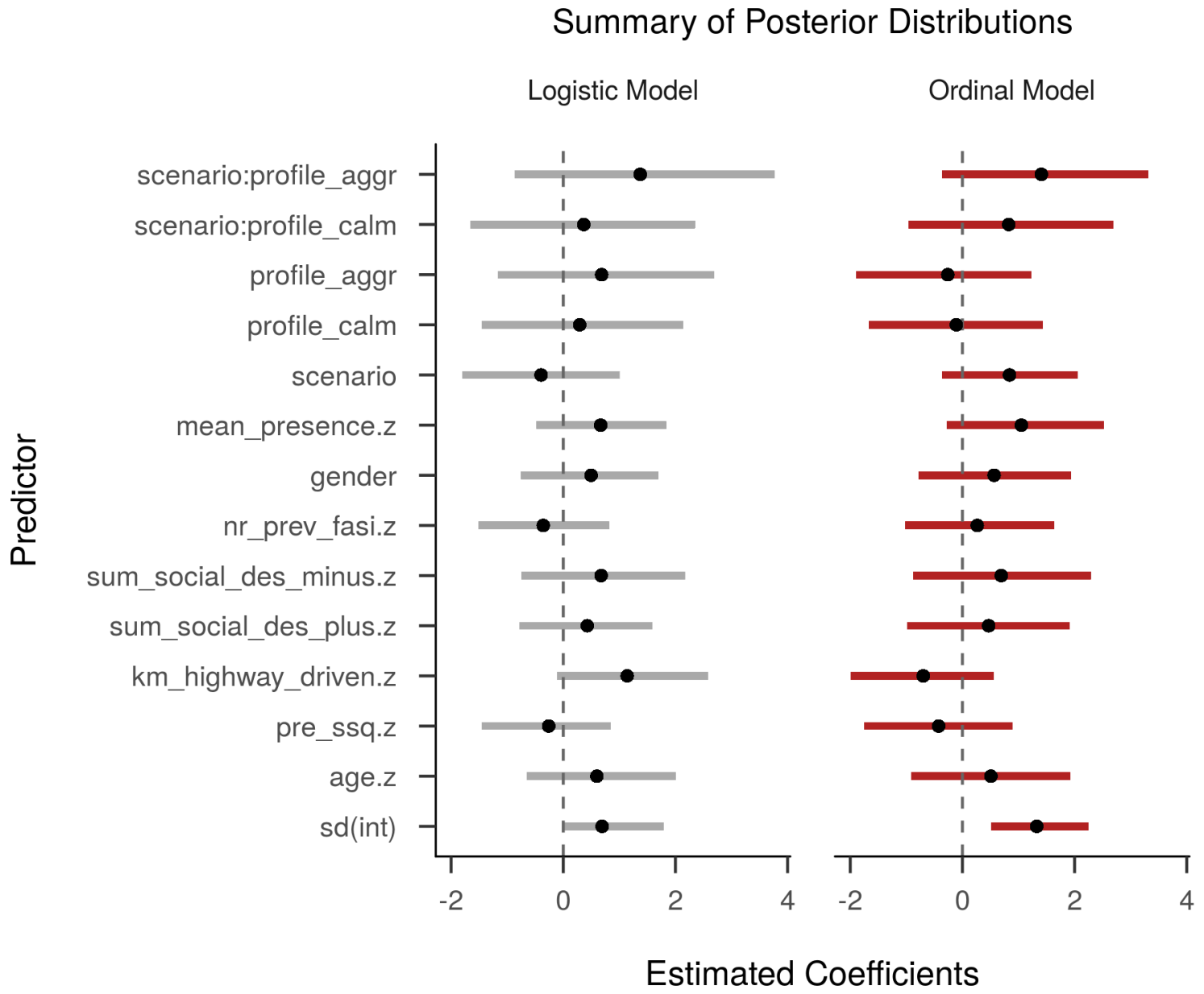

FIGURE 3.6: Shows the mean and 95\% credible intervals for the posterior distribution of predictors in the full logistic and full ordinal regression models, respectively. Note that continuous predictors have been scaled by subtracting the mean and dividing by twice the standard deviation (Gelman, 2008). profile_aggr, profile_calm, and scenario are dummy coded variables indicating the conditions; mean_presence. $z$ is the average score in the presence questionnaire; gender is a dummy coded variable indicating gender $(0=$ female $)$; sum_social...z are sums of the two social desirability subscales; $k m \_h i g h w a y \_d r i v e n . z$ are the kilometers that were driven on highways the previous year; pre_ssq.z is the simulator sickness score in the preliminary interview; $s d($ int $)$ is the standard deviation of the participant-specific offsets to the intercept.

patterns, see Figure 3.7. However, because each participant ascended onto the highway only once, the number of responses for this scenario is only about a third of the overtaking scenarios, with only 10, 11, and 11 response for the aggressive, calm, and old driver profiles, respectively. With these few data points, it seems premature to draw any conclusions from 
Proportion judged Human

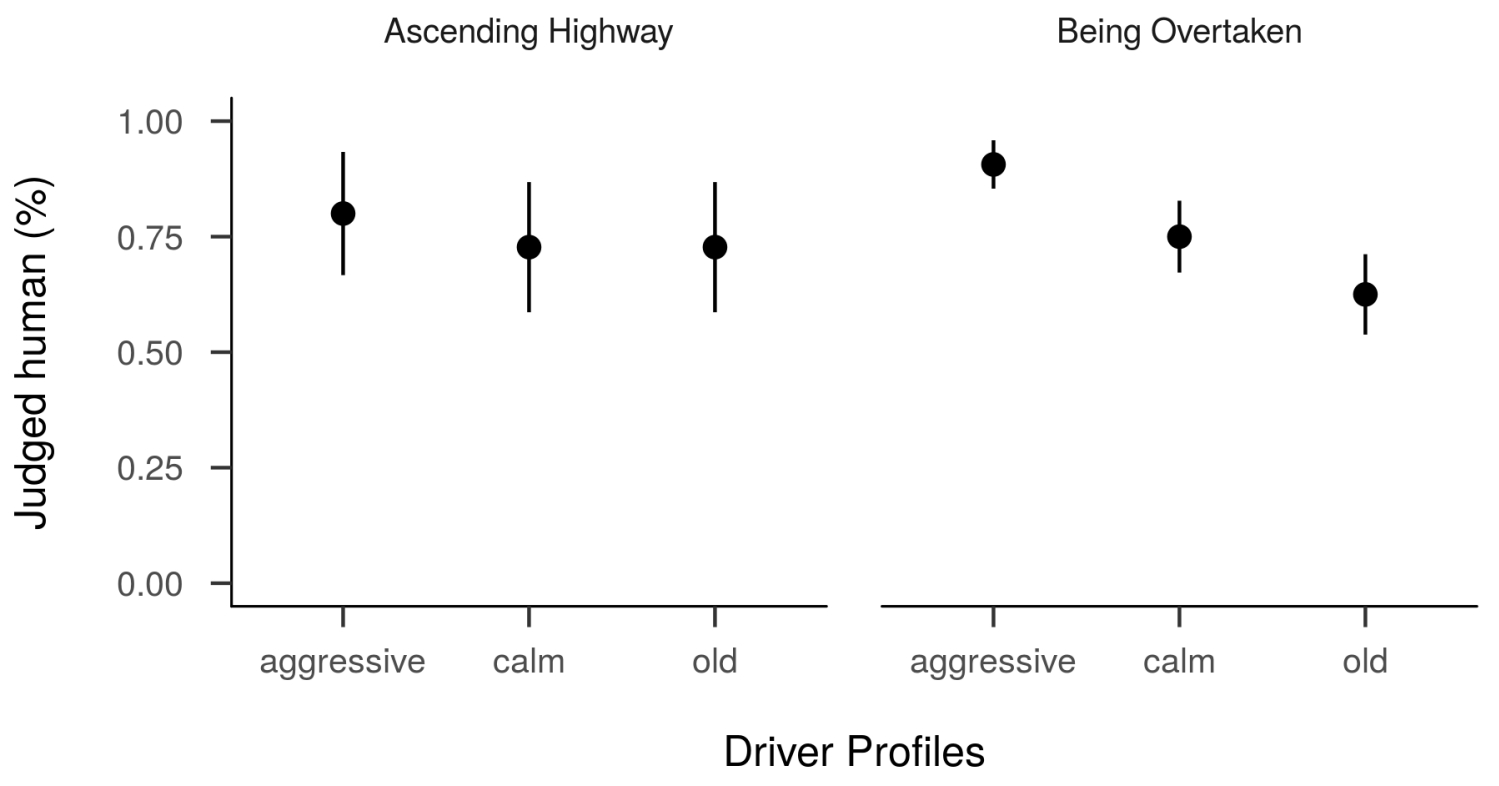

FIGURE 3.7: Shows mean and standard deviation of the proportion judged human for the respective driver profiles for each scenario.

this differential pattern. Speculation suggests, though, that this might have occurred because speed is less pronounced when one merges before a car compared to when the same car overtakes oneself. In the overtaking scenarios, people were likely to judge the car as human when it drove faster than they expected, thinking — as their stated in the post-experimental interviews - that a computer would neither violate traffic rules nor norms. When ascending onto the highway, the speed of the other car might have been less pronounced and thus the aggressive driver was not judged as frequently as being human. This points to the fact that one needs to carefully consider the traffic scenarios in which comparisons are made.

No control variable, which includes presence, social desirability, the number of kilometres driven during the last year, the simulator sickness score before the driving, sex, and age, seems to exhibit a strong effect on the dependent variables. 


\subsubsection{Qualitative Results}

In the last fifteen minutes of the experiment, participants were driving freely on the highway with a maximum speed of $120 \mathrm{~km} / \mathrm{h}$. Traffic around them included all six driver profiles. 36 participants completed this part. They were asked to think aloud during the whole segment, commenting on anything that seems unrealistic as well as realistic.

There is no straightforward way to encode time in the qualitative interpretation. The think aloud protocol took about fifteen minutes, and in fifteen minutes a lot can happen; traffic could be realistic at the start, and become progressively more unrealistic. Most participants commented that some aspects were realistic, while other parts were not. Which aspects were unrealistic?

The majority of participants mentioned the low traffic density. There were periods when there was no traffic at all, and this greatly reduced immersion. Additionally, several participants mentioned that, overall, the driving behaviour of the other drivers was too safe - no dangerous situations occurred, and this led some of the participants to believe that the other drivers were computer generated. In general, other drivers (a) held their lane too perfectly, never fluctuating within the lane, (b) held unrealistically large headways to the ego as well as to other drivers, (c) always used their indicator, which, although lawful, is unrealistic for real driving behaviour, (d) never created uneasy or dangerous situations, (e) frequently took their obligation to drive on the right too seriously, which sometimes led to awkward overtaking situations (overtaking, shifting back to the right lane, overtaking, shifting back to the right lane, etc.). Moreover, the trucks were too homogeneous in holding headway constant, and too infrequently overtook one another. Sometimes the other cars slowed their speed down with no apparent reason. With respect to the environment, one participant noted that there were no roadworks which is, however, quite usual on the real road. Additionally, one participant noted that the license plates all were from around the area (Stuttgart) which she felt was also unrealistic. 
Based on these insights, I suggest the following points to improve the simulation: Increase traffic density. There should not be (extensive) periods without traffic. Decrease the headway between drivers, and make it more variable. Have other drivers fluctuate on their lane, instead of having them drive perfectly. Decrease the amount of indicating. Increase the probability of trucks overtaking each other. Overall, increase the amount of dangerous traffic situations. There should be more frequently situations in which the driver feels unease and has to increase their attention. As several participants put it, there should be an increase in "Raser und Drängler"'.

Overall, however, participants felt that the driving simulation was realistic, and the majority of the participants enjoyed the experience.

\subsection{Discussion}

An important aspect of validity in driving simulators is how well computer-generated agents mimic real driving behaviour. This aspect has been mostly ignored in the literature, which focuses on absolute and relative behavioural validity. In this study, we amended this shortcoming by taking first steps towards validating computer-generated agents that are modeled based on empirical driver profiles. Participants had to judge whether an aggressive or a calm driver behaves in a way that more closely resembles human driving as well as being overall more realistic compared to computer-generated agents that did not have a distinct driver profile. Using confirmatory, directed Bayesian tests we found evidence for a stronger resemblance to human driving only for the aggressive driver profile; for the realism judgements, the data were uninformative. By conducting this experiment, we have gained a number of insights not only about the traffic simulation but also on what is required for future studies to improve their informativity.

\footnotetext{
${ }^{9}$ These are things that the Daimler team has already implemented since the experiment, or is currently working on.
} 
First, our sample size, consisting of only 32 participants, is comparatively low for a driving simulator study. We partially mitigated this problem by using weakly informative priors and testing directed hypotheses, both of which made our inferences stronger by incorporating sensible prior knowledge. Future studies, however, must collect more data. One way to do so, despite simply increasing the sample size, is to shorten the qualitative, and increase the quantitative part of the experiment. In the current experiment, we have used only about twelve minutes for the experimental part, and fifteen for the qualitative, exploratory part. In retrospect, one could have increased the experimental part by another overtaking scenario, and future studies should try to rebalance; at some point in the qualitative part, participants tend to repeat themselves and information gain lapses.

Second, we focused on overtaking scenarios but included ascending onto the highway because participants had to ascend onto the highway anyway. Even though the analysis including the difference between those two scenarios was exploratory and we had only few data points for the ascending scenario, we found that judgements for those two situations tended to differ. This is an important reminder that traffic simulations can only be validated with respect to certain scenarios, and never as a whole. Future endeavours should compile a list of scenarios that are routinely used in driving simulator studies. Those scenarios can then be validated piece by piece. They will differ in what features of the driver profiles become apparent, and this will most likely influence participants' judgements. For example, as became apparent in the qualitative analysis, participants thought that drivers which drove much faster compared to one's own speed were more likely to be humans than computers; computers, so the argument, would conform more strongly to rules and norms. In some scenarios, speed will be a pronounced feature — such as in overtaking scenarios - whereas in others it might play only a secondary role - for example, when two lanes narrow into one, and one waits until a driver allows one to merge; here, politeness plays a much bigger role. 
Third, and related to the points above, the overtaking situations might not have been critical enough to distinguish reliably between drivers. Recall that participants had to drive on the rightmost lane and were subsequently overtaken by a car with a certain driver profile. However, because we wanted the situation to be as controlled as possible, only two cars, including the participant's, featured in each scenario. This led to the other car having lots of space for the overtaking maneouvre. It could veer to the middle lane far before the ego vehicle, and go back to the right lane long after it has passed the ego vehicle. Thus, the only distinguishing features were whether the car went back to the right lane or stayed in the middle one - and speed. Future studies should carefully think about the tradeoff between controlled and naturalistic situations. Having more cars on the lane, with only the driver profile under scrutiny really engaging, also leads to increased situations in which the participant can observe the driver profile interacting with other cars, yielding more information on which the participant's judgement is ultimately based. This increase in information might also reduce the variability in interpreting the questions, which leads us to the next point.

Fourth, future experiments need to carefully think about measurement. Some participants, for example, had difficulties with the distinction between artificial and human driving behaviour especially in the first part of the experiment in which highly structured manoeuvres took place. For example, most participants stated that artificial drivers would conform to the norms and rules of driving; would make no errors; would drive foresighted; and would engage neither in speeding nor in tailgating. However, humans can behave this way, too, and this made it difficult for some participants to judge whether the car was driven by a human or a computer. This could be in part to the fact that these manoeuvres were rather short; participants generated less equivocal statements in the last segment of the experiment which features multiple vehicles. This suggests constructing more natural scenarios, as mentioned above.

These issues with the binary "human or computer" judgements carry over to ordinal judgements of realism, too. There is no gold standard, no precise conditions for when a driving 
behaviour is realistic. For example, most participants noted the lawful indicating of other drivers. Some of them viewed this behaviour as being computer-generated - most people do not indicate on the highway - while others viewed it as being realistic. The same circumstances, then, caused different people to come to opposite conclusions. This indicates that realism is far from a unidimensional construct, but is instead the result of a number of considerations which differ across participants. Thus, future studies should reconsider the, on the surface, unproblematic realism question and ask participants more specific questions.

The points above are substantiated by the somewhat counterintuitive finding that the binary judgements did provide more information than the ordinal realism ratings. This is indicated by the difference in the respective Bayes factors: while we found moderate to strong evidence that, for example, aggressive drivers were more frequently perceived as being human, the data was equivocal with respect to the realism judgements.

They also point to variability both in terms of driving behaviour exhibited across scenarios, as well as to variability in the extent to which participants interpret it as being realistic. It might be that the variance in actual driving behaviour is much larger than the variance between the driving behaviour of humans and computers, which would make it difficult to distinguish the two in actual driving. A related point is the extent to which the six driver profiles overlap in different scenarios such as ascending onto the highway or overtaking other cars. A future study could look into this by creating simulated scenarios in which each driver profile, respectively, plays the pivotal role. Providing participants with a description of the six driver profiles, they could then be asked to rate which profile was most likely to exhibit the behaviour displayed; or asked to make binary judgements in a forced-choice paradigm. The results could be clustered with multidimensional scaling, which would indicate the overlap between driver profiles. Such a study could even be conducted online, accessing a much larger group of people than possible with driving simulator studies.

The qualitative part generated a number of insights that are currently being implemented. Interestingly, the majority of participants stated that there were too few "Raser und Drängler" 
in the simulation; their occurrence in the real world is much higher. There are two interpretations to this. First, note that there were no dangerous situations in the traffic simulation; participants never experienced other drivers abruptly changing lanes, which would have required immediate action in order to avoid an accident. While such dangerous situations might occurre only rarely even on the real road - rendering it unlikely that they occurre in this particular fifteen minutes of highway driving in the simulation - they do occurre sometimes, and participants might both overestimate their frequency (Kahneman \& Tversky, 1979), as well as arrive at the simulator with the expectation of a dangerous situation happening. In fact, some participants even stated in the interview that, because traffic was so peaceful, they expected a dangerous situation lurking. To create such dangerous situations, the traffic simulation could be extended by including emotion parameters, such as anger, that charge themselves while driving and which, at some point, result in reckless behaviour. A second, related interpretation of the statement that there were too few "Raser und Dränger" is that, in fact, assumptions of the Daimler traffic model could be wrong. The traffic model assumes six distinct driver profiles based on empirical studies conducted in the 1970s, 1980s and 1990s (Hürlimann, 1996, p. 67-106) which estimated the occurrence of affective and aggressive drivers - those that have the potential to create dangerous situations — at only $10 \%$ and $6 \%$, respectively. These estimates might well be out of date.

Considering the seniority of the empirical studies leads us back to measurement: what does it mean to ask whether the car was driven by a human or a computer? What, in 2017, with all the driver assistance gadgets and semi-autonomous driving functions, does it even mean for driving behaviour to be human? Above I have argued that there might be too few aggressive drivers in the simulation. There is, however, an association between aggressive driving and car accidents (Hürlimann, 1996), and this is undesirable. One of the strongest arguments in favour of autonomous driving, by many heralded as an inevitable future, is exactly the reduction of traffic accidents, which implies a decrease in aggressive driving. Thus, in the not too distant future, autonomous driving will increase homogeneity in driving behaviour. 
Could it be, then, that efforts to create variability between computer-generated agents is betting on the wrong horse, considering the future? I do not think so. First, it is unclear when autonomous driving will be the status quo or what its implications will be exactly; modeling inter-individual variability in driving behaviour will be crucial even beyond this timepoint, considering the age of our society, and its slow adoption of new technologies. However, the point about what it means for driving behaviour to be human still remains, especially on the highway, where semi-autonomous driving functions are already available. While these functions do increase homogeneity between drivers - after all, it is the same computer driving, whether the human is otherwise an aggressive or calm driver — there will still, I suspect, be differences among drivers on the road; they will just be less pronounced. For the time being, then, modeling inter-individual differences in driving behaviour remains important.

Fifth, strictly speaking, we looked at incremental validity: do the new driver profiles improve upon the older versions? An ideal experimental procedure would have access to "ground truth", comparing the differences between driver profiles against human drivers. Although incremental validity is desirable, showing that the new driver profiles are judged as being equally good as actual human drivers is the more relevant endeavour. Such a design could be achieved by either having humans drive a car in the driving simulator, or use real driving data and convert it into simulated trajectories of the cars. Computer-generated behaviour can than be compared against this human driving behaviour.

Finally, most of the participants have already participated in a driving simulator study three or more times. However, it is unclear whether this is a limitation or strength of the current study. On the one hand, one could argue that participants with previous experience in driving simulators have the preconceived notion that traffic is not realistic. On the other hand, this experience could mean that they look more closely at potential mismatches with reality, and provide more accurate judgements. In any case, future studies should be aware of the previous experience of participants and make informed choices. 
To sum up, we have proposed a novel experimental design to evaluate how participants perceive computer-generated drivers which have dedicated, psychologically informed driving styles. We have used informed, Bayesian hierarchical regression models to test our directed hypotheses, provided an extensive post-mortem analysis of the experiment, as well as suggested directions for further research. 


\section{References}

Anderson, E. (1936). The species problem in Iris. Annals of the Missouri Botanical Garden, 23(3), 457-509. JSTOR: 2394164

Baayen, H., Davidson, D. J., \& Bates, D. (2008). Mixed-effects modeling with crossed random effects for subjects and items. Journal of Memory and Language, 59(4), 390412. doi:10.1016/j.jml.2007.12.005

Barr, D. J., Levy, R., Scheepers, C., \& Tily, H. J. (2013). Random effects structure for confirmatory hypothesis testing: Keep it maximal. Journal of Memory and Language, 68(3), 255-278. doi:10.1016/j.jml.2012.11.001

Bates, D., Kliegl, R., Vasishth, S., \& Baayen, H. (2015). Parsimonious mixed models. arXiv preprint arXiv:1506.04967. Retrieved from https://arxiv.org/abs/1506.04967

Baumann, G., Riemer, T., Liedecke, C., Rumbolz, P., \& Schmidt, A. (2012). How to build Europe's largest eight-axes motion simulator. In Proceedings of the driving simulation conference.

Berger, J. O. \& Berry, D. A. (1988). Statistical analysis and the illusion of objectivity. American Scientist, 76(2), 159-165. JSTOR: 27855070

Berkson, J. (1938). Some difficulties of interpretation encountered in the application of the chi-square test. Journal of the American Statistical Association, 33(203), 526-536.

Breiman, L. (2001). Random forests. Machine learning, 45(1), 5-32.

Brooks, J. O., Goodenough, R. R., Crisler, M. C., Klein, N. D., Alley, R. L., Koon, B. L., ... Wills, R. F. (2010). Simulator sickness during driving simulation studies. Accident Analysis \& Prevention, 42(3), 788-796. doi:10.1016/j.aap.2009.04.013 
Bürkner, P.-C. (2017). Brms: An R Package for Bayesian Multilevel Models using Stan. Journal of Statistical Software.

Carpenter, B., Gelman, A., Hoffman, M., Lee, D., Goodrich, B., Betancourt, M., ... Riddell, A. (2017). Stan: A probabilistic programming language. Journal of Statistical Software, 76(1), 1-32. doi:10.18637/jss.v076.i01

Carsten, O. \& Jamson, A. H. (2011). Driving simulators as research tools in traffic psychology. Handbook of traffic psychology, 87-96.

Cohen, J. (1968). Multiple regression as a general data-analytic system. Psychological Bulletin, 70(6), 426-443. doi:10.1037/h0026714

Cristea, R., Rulewitz, S., Radusch, I., Hübner, K., \& Schünemann, B. (2016). Implementation of Cognitive Driver Models in Microscopic Traffic Simulations. In Proceedings of the 9th EAI International Conference on Simulation Tools and Techniques (pp. 104111). ICST (Institute for Computer Sciences, Social-Informatics and Telecommunications Engineering).

Cutler, D. R., Edwards, T. C., Beard, K. H., Cutler, A., Hess, K. T., Gibson, J., \& Lawler, J. J. (2007). Random forests for classification in ecology. Ecology, 88(11), 2783-2792. doi:10.1890/07-0539.1

De Winter, J., Van Leuween, P., \& Happee, P. (2012). Advantages and disadvantages of driving simulators: A discussion. In Proceedings of Measuring Behavior (pp. 47-50). Citeseer. Retrieved January 13, 2017, from https://goo.gl/xbWbuC

DeGroot, M. H. (1982). Lindley's paradox: Comment. Journal of the American Statistical Association, 77(378), 336-339.

Efron, B. \& Hastie, T. (2016). Computer Age Statistical Inference. Cambridge University Press.

Efron, B. \& Morris, C. (1977). Stein's Paradox in Statistics. Scientific American, 119-127.

Etz, A., Gronau, Q. F., Dablander, F., Edelsbrunner, P. A., \& Baribault, B. (2017). How to become a Bayesian in eight easy steps: An annotated reading list. Psychonomic Bulletin \& Review, 1-16. doi:10.3758/s13423-017-1317-5 
Fisher, D. L., Rizzo, M., Caird, J., \& Lee, J. D. (2011). Handbook of driving simulation for engineering, medicine, and psychology. CRC Press.

Fisher, R. A. (1936). The use of multiple measurements in taxonomic problems. Annals of Eugenics, 7(2), 179-188. doi:10.1111/j.1469-1809.1936.tb02137.x

Flade, A. \& Limbourg, M. (1999). Frauen und Männer in der mobilen Gesellschaft. Springer.

Follmer, R., Gruschwitz, D., Jesske, B., Quandt, S., Lenz, B., Nobis, C., ... Mehlin, M. (2010). Mobilität in Deutschland 2008. Methodenbericht. Bonn und Berlin.

Gelman, A. (2006). Multilevel (hierarchical) modeling: what it can and cannot do. Technometrics, 48(3), 432-435. doi:10.1198/004017005000000661

Gelman, A. (2008). Scaling regression inputs by dividing by two standard deviations. Statistics in Medicine, 27(15), 2865-2873. doi:10.1002/sim.3107

Gelman, A., Jakulin, A., Pittau, M. G., \& Su, Y.-S. (2008). A weakly informative default prior distribution for logistic and other regression models. The Annals of Applied Statistics, 1360-1383. doi:10.1214/08-AOAS191

Gelman, A. \& Rubin, D. B. (1992). Inference from iterative simulation using multiple sequences. Statistical science, 7(4), 457-472.

Gini, C. (1912). Variabilità e mutabilità. Reprinted in Memorie di metodologica statistica (Ed. Pizetti E, Salvemini, T). Rome: Libreria Eredi Virgilio Veschi.

Guest, O. (2017, February 26). Using the Gini Coefficient to Evaluate Deep Neural Network Layer Representations. Retrieved June 29, 2017, from http://neuroplausible.com/gini

Hale, K. S., Stanney, K. M., Keshavarz, B., Hecht, H., \& Lawson, B. D. (2014). Visually induced motion sickness: causes, characteristics, and countermeasures. In Handbook of Virtual Environments: Design, Implementation, and Applications, Second Edition (pp. 647-698). CRC Press.

Haller, H. \& Krauss, S. (2002). Misinterpretations of significance: A problem students share with their teachers. Methods of Psychological Research, 7(1), 1-20.

Hastie, T., Tibshirani, R., \& Friedman, J. (2009). The Elements of Statistical Learning. New York: Springer. 
Heathcote, A., Brown, S. D., \& Wagenmakers, E.-J. (2015). An introduction to good practices in cognitive modeling. In An Introduction to Model-Based Cognitive Neuroscience (pp. 25-48). Springer.

Helbing, D., Hennecke, A., Shvetsov, V., \& Treiber, M. (2001). MASTER: macroscopic traffic simulation based on a gas-kinetic, non-local traffic model. Transportation Research Part B: Methodological, 35(2), 183-211. doi:10.1016/S0191-2615(99)00047-8

Helland, A., Lydersen, S., Lervåg, L.-E., Jenssen, G. D., Mørland, J., \& Slørdal, L. (2016, September). Driving simulator sickness: Impact on driving performance, influence of blood alcohol concentration, and effect of repeated simulator exposures. Accident Analysis \& Prevention, 94, 180-187. doi:10.1016/j.aap.2016.05.008

Hennig, C. (2015). What are the true clusters? Pattern Recognition Letters, 64, 53-62. doi:10.1016/j.patrec.2015.04.009

Hoekstra, R., Morey, R. D., Rouder, J. N., \& Wagenmakers, E.-J. (2014). Robust misinterpretation of confidence intervals. Psychonomic Bulletin \& Review, 21(5), 1157-1164. doi:10.3758/s13423-013-0572-3

Hout, M. C., Papesh, M. H., \& Goldinger, S. D. (2013). Multidimensional scaling. Wiley Interdisciplinary Reviews: Cognitive Science, 4(1), 93-103. doi:10.1002/wcs.1203

Hürlimann, F. W. (1996). Typologie und Verkehr. Zürich: Verlag Heinrich Vogel.

Jackman, S. (2009). Bayesian Analysis for the Social Sciences. John Wiley \& Sons.

Jaeger, T. F. (2008). Categorical data analysis: Away from ANOVAs (transformation or not) and towards logit mixed models. Journal of Memory and Language, 59(4), 434-446. doi:10.1016/j.jml.2007.11.007

Jöri, H. (2002). Ruhig bis aggressiv-Sechs Typen am Steuer. punktum. Zeitschrift des Schweizerischen Berufsverbands für Angewandte Psychologie, 18-19. Retrieved from https: //goo.g1/6tcxg7

Kahneman, D. \& Tversky, A. (1979). Prospect Theory: An Analysis of Decision under Risk. Econometrica, 47(2), 263-291. doi:10.2307/1914185 


\section{REFERENCES}

Kass, R. E. \& Raftery, A. E. (1995). Bayes factors. Journal of the American Statistical Association, 90(430), 773-795.

Kemper, C., Beierlein, C., Bensch, D., Kovaleva, A., \& Rammstedt, B. (2012). Eine Kurzskala zur Erfassung des Gamma-Faktors sozial erwünschten Antwortverhaltens: Die Kurzskala Soziale Erwünschtheit-Gamma (KSE-G). GESIS. Retrieved from https ://goo.gl/ Ams4qK

Kennedy, R. S., Lane, N. E., Berbaum, K. S., \& Lilienthal, M. G. (1993). Simulator sickness questionnaire: An enhanced method for quantifying simulator sickness. The International Journal of Aviation Psychology, 3(3), 203-220. doi:10.1207/s15327108ijap0303_ 3

Keshavarz, B. \& Hecht, H. (2011). Validating an efficient method to quantify motion sickness. Human Factors, 53(4), 415-426. doi:10.1177/0018720811403736

Kesting, A., Treiber, M., \& Helbing, D. (2007). General lane-changing model MOBIL for car-following models. Transportation Research Record: Journal of the Transportation Research Board. doi:10.3141/1999-10

Kesting, A., Treiber, M., \& Helbing, D. (2009). Agents for Traffic Simulation. Multi-Agent Systems, 325. doi:10.1201/9781420070248.ch11

Klugkist, I. \& Hoijtink, H. (2007). The Bayes factor for inequality and about equality constrained models. Computational Statistics \& Data Analysis, 51(12), 6367-6379. doi:10.1016/j.csda.2007.01.024

Klüver, M., Herrigel, C., Heinrich, C., Schöner, H.-P., \& Hecht, H. (2016). The behavioral validity of dual-task driving performance in fixed and moving base driving simulators. Transportation Research Part F: Traffic Psychology and Behaviour, 37, 78-96. doi:10. 1016/j.trf.2015.12.005

Klüver, M., Herrigel, C., Preuß, S., Schöner, H.-P., \& Hecht, H. (2012). Comparing the Incidence of Simulator Sickness in Five Different Driving Simulators. In Driving Simulation Conference 2015 (pp. 1-8). Tübingen. 
Kruschke, J. K. \& Liddell, T. M. (2017). The Bayesian New Statistics: Hypothesis testing, estimation, meta-analysis, and power analysis from a Bayesian perspective. Psychonomic Bulletin \& Review, 1-29. doi:10.3758/s13423-016-1221-4

Lecoutre, M.-P., Poitevineau, J., \& Lecoutre, B. (2003). Even statisticians are not immune to misinterpretations of Null Hypothesis Significance Tests. International Journal of Psychology, 38(1), 37-45. doi:10.1080/00207590244000250

Liang, F., Paulo, R., Molina, G., Clyde, M. A., \& Berger, J. O. (2008). Mixtures of g priors for Bayesian variable selection. Journal of the American Statistical Association, 103(481), 410-423. doi:10.1198/016214507000001337

Liaw, A. \& Wiener, M. (2002). Classification and regression by randomForest. $R$ news, 2(3), $18-22$.

Lindley, D. V. (1957). A statistical paradox. Biometrika, 44(1), 187-192.

Lindley, D. V. (2000). The philosophy of statistics. Journal of the Royal Statistical Society: Series D (The Statistician), 49(3), 293-337.

Loh, W.-Y. (2011). Classification and regression trees. Wiley Interdisciplinary Reviews: Data Mining and Knowledge Discovery, 1(1), 14-23. doi:10.1002/widm.8

Matuschek, H., Kliegl, R., Vasishth, S., Baayen, H., \& Bates, D. (2017). Balancing Type I error and power in linear mixed models. Journal of Memory and Language, 94, 305315. doi:10.1016/j.jml.2017.01.001

McElreath, R. (2016). Statistical rethinking: A Bayesian course with examples in R and Stan. CRC Press.

McGrayne, S. B. (2011). The Theory That Would Not Die: How Bayes' Rule Cracked the Enigma Code, Hunted Down Russian Submarines, and Emerged Triumphant from Two Centuries of Controversy. Yale University Press.

McNeish, D. (2016). On using Bayesian methods to address small sample problems. Structural Equation Modeling: A Multidisciplinary Journal, 23(5), 750-773. doi:10.1080/ 10705511.2016.1186549 
Menze, B. H., Kelm, B. M., Masuch, R., Himmelreich, U., Bachert, P., Petrich, W., \& Hamprecht, F. A. (2009). A comparison of random forest and its Gini importance with standard chemometric methods for the feature selection and classification of spectral data. BMC Bioinformatics, 10(1), 213. doi:10.1186/1471-2105-10-213

Morey, R. D., Romeijn, J.-W., \& Rouder, J. N. (2013). The humble Bayesian: model checking from a fully Bayesian perspective. British Journal of Mathematical and Statistical Psychology, 66(1), 68-75. doi:10.1111/j.2044-8317.2012.02067.x

Morey, R. D., Romeijn, J.-W., \& Rouder, J. N. (2016). The philosophy of Bayes factors and the quantification of statistical evidence. Journal of Mathematical Psychology, 72, 618. doi:10.1016/j.jmp.2015.11.001

Nourzad, S. H. H., Salvucci, D. D., \& Pradhan, A. (2014). Computational Modeling of Driver Distraction by Integrating Cognitive and Agent-based Traffic Simulation Models. In Computing in Civil and Building Engineering (2014) (pp. 1885-1892).

Oakes, M. (1986). Statistical inference: A commentary for the social and behavioral sciences. New York: Wiley.

Platt, J. R. (1964). Strong inference. Science, 146(3642), 347-353.

Poline, J.-B. \& Brett, M. (2012). The general linear model and fMRI: does love last forever? Neuroimage, 62(2), 871-880. doi:10.1016/j.neuroimage.2012.01.133

R Core Team. (2017). R: A Language and Environment for Statistical Computing. Vienna, Austria: R Foundation for Statistical Computing. Retrieved from https://www. R project.org/

Reason, J. T. \& Brand, J. J. (1975). Motion Sickness. Cambridge, Massachusetts: Academic press.

Rouder, J. N. \& Morey, R. D. (2012). Default Bayes factors for model selection in regression. Multivariate Behavioral Research, 47(6), 877-903. doi:10.1080/00273171.2012. 734737 
Rouder, J. N., Morey, R. D., Verhagen, J., Province, J. M., \& Wagenmakers, E.-J. (2016). Is there a free lunch in inference? Topics in Cognitive Science, 8(3), 520-547. doi:10. 1111/tops. 12214

Rouder, J. N., Speckman, P. L., Sun, D., Morey, R. D., \& Iverson, G. (2009, April 1). Bayesian $\mathrm{t}$ tests for accepting and rejecting the null hypothesis. Psychonomic Bulletin \& Review, 16(2), 225-237. doi:10.3758/PBR.16.2.225

Rouder, J., Morey, R., \& Wagenmakers, E.-J. (2016). The interplay between subjectivity, statistical practice, and psychological science. Collabra: Psychology, 2(1). doi:10 . 1525/collabra.28

Schöner, H.-P. \& Morys, B. (2015). Dynamische Fahrsimulatoren. In Handbuch Fahrerassistenzsysteme (pp. 139-154). Springer.

Slater, M., Usoh, M., \& Steed, A. (1995). Taking steps: the influence of a walking technique on presence in virtual reality. ACM Transactions on Computer-Human Interaction (TOCHI), 2(3), 201-219.

Sorensen, T. \& Vasishth, S. (2015). Bayesian linear mixed models using Stan: a tutorial for psychologists, linguists, and cognitive scientists. arXiv preprint arXiv:1506.06201.

Stevens, S. S. (1946). On the Theory of Scales of Measurement. Science, 103(2684), 677680.

Steyvers, M. (2002). Multidimensional scaling. Encyclopedia of cognitive science.

Strobl, C., Malley, J., \& Tutz, G. (2009). An introduction to recursive partitioning: rationale, application, and characteristics of classification and regression trees, bagging, and random forests. Psychological Methods, 14(4), 323. doi:10.1037/a0016973

Treiber, M., Hennecke, A., \& Helbing, D. (2000). Congested traffic states in empirical observations and microscopic simulations. Physical Review E, 62(2), 1805. doi:10.1103/ PhysRevE.62.1805

van Wageningen-Kessels, F., Van Lint, H., Vuik, K., \& Hoogendoorn, S. (2015). Genealogy of traffic flow models. EURO Journal on Transportation and Logistics, 4(4), 445-473. doi:10.1007/s13676-014-0045-5 


\section{REFERENCES}

Vandekerckhove, J., Matzke, D., \& Wagenmakers, E.-J. (2015). Model comparison and the principle of parsimony. In Oxford Handbook of Computational and Mathematical Psychology (pp. 300-320). Oxford: Oxford University Press.

Wagenmakers, E.-J. (2007). A practical solution to the pervasive problems of p values. Psychonomic Bulletin \& Review, 14(5), 779-804. doi:10.3758/BF03194105

Wagenmakers, E.-J., Lodewyckx, T., Kuriyal, H., \& Grasman, R. (2010). Bayesian hypothesis testing for psychologists: A tutorial on the Savage-Dickey method. Cognitive Psychology, 60(3), 158-189. doi:10.1016/j.cogpsych.2009.12.001

Wetzels, R., Grasman, R. P. P. P., \& Wagenmakers, E.-J. (2010). An encompassing prior generalization of the Savage-Dickey density ratio. Computational Statistics \& Data Analysis, 54(9), 2094-2102. doi:10.1016/j.csda.2010.03.016

Wickelmaier, F. (2003). An introduction to MDS. Sound Quality Research Unit, Aalborg University, Denmark, 46. Retrieved from http://homepages.uni-tuebingen.de/florian. wickelmaier/pubs/Wickelmaier2003SQRU.pdf

Wickham, H. (2014). Tidy data. Journal of Statistical Software, 59(10), 1-23. doi:10.18637/ jss.v059.i10

Wulff, H. R., Andersen, B., Brandenhoff, P., \& Guttler, F. (1987). What do doctors know about statistics? Statistics in Medicine, 6(1), 3-10. doi:10.1002/sim.4780060103 